\title{
Robust Fast Adaptive Fault Estimation for T-S Fuzzy Markovian Jumping Systems with Mode-Dependent Time-Varying State Delays
}

\author{
Chao Sun $\mathbb{D}^{1},{ }^{1}$ Shengjuan Huang, ${ }^{1}$ Libing $W u,{ }^{1}$ and Suhuan $Y i \mathbb{D}^{2}$ \\ ${ }^{1}$ School of Science, University of Science and Technology Liaoning, Anshan 114051, China \\ ${ }^{2}$ School of Applied Technology, University of Science and Technology Liaoning, Anshan 114051, China \\ Correspondence should be addressed to Suhuan Yi; 40108454@qq.com
}

Received 26 October 2020; Revised 11 February 2021; Accepted 2 March 2021; Published 22 March 2021

Academic Editor: Nicolae Herisanu

Copyright (c) 2021 Chao Sun et al. This is an open access article distributed under the Creative Commons Attribution License, which permits unrestricted use, distribution, and reproduction in any medium, provided the original work is properly cited.

\begin{abstract}
This paper studies the problem of actuator fault estimation for a class of T-S fuzzy Markovian jumping systems, which is subject to mode-dependent interval time-varying delays and norm-bounded external disturbance. Based on the given fast adaptive estimation algorithm and by employing a novel Lyapunov-Krasovskii function candidate, a robust fault estimation scheme is proposed to estimate faults whose derivative is bounded. With this improved method, the proposed fault estimator minimizes the effect of disturbance on the estimation error and reduces the conservatism of systems stability results simultaneously. To be specific, an improved mode-dependent criterion for the existence of the fault estimation observer is established to guarantee the error dynamic system to be stochastically stable with a prescribed $H_{\infty}$ performance and reduce the conservatism of designing procedure. Finally, three numerical examples are given to show the effectiveness of the proposed method.
\end{abstract}

\section{Introduction}

As an important class of stochastic systems, Markovian jump systems (MJSs) have been widely concerned over the past several decades. This kind of system model can be utilized to represent many types of physical systems in applications, such as electric power systems [1], manufacturing systems [2], and circuit systems [3]. The study of MJSs has been of both theoretical and practical importance, and many results have been reported in the literatures [4-6]. However, the existing results are mainly about the linear MJSs; little attention has been paid to nonlinear MJSs. Recent years have witnessed the growing interests of the Takagi-Sugeno (T-S) fuzzy model [7]. Extensive research suggests that the T-S fuzzy model can provide a general framework to approximate the nonlinear system, such as stochastic systems and hybrid systems. Therefore, it is natural that the nonlinear MJSs are represented by T-S fuzzy modes which can be described by the fuzzy IF-THEN rules. So far, under the framework of fuzzy logic control strategy, considerable attention has been paid to the stability analysis and controller synthesis for MJSs, such as robust filtering $[8,9]$ and $H_{\infty}$ control [10-13]. Recently, it is worth mentioning that, in [12], with the aid of a mode-dependent Lyapunov-Krasovskii functional and the Abel lemma-based finite sum inequality, the finite-time bounded control problem has been solved for a class of discrete-time T-S fuzzy Markov jump systems with memory state feedback controller. By exploiting Lyapunov stability theory and Wirtinger-based integral inequalities, literature [13] deals with the robust trajectory tracking control problem for T-S fuzzy Markovian jump systems with time-varying delay, uncertainties, and disturbances.

On another research front line, due to the more increasing demand for reliability and safety in industrial control processes, the issues of fault detection and isolation (FDI), fault-tolerant control algorithms, and their applications to a wide range of industrial processes have been the subjects of intensive investigation in the recent years. For instance, by using adaptive backstepping technique, the 
work of Wang et al. [14] proposes a fault-tolerant compensation control approach against nonlinearity and simultaneous additive and multiplicative actuator faults. In [15], the fault-tolerant control problem against sensor faults of a time-delay Markov jump systems with Itô stochastic and output disturbances has been investigated by using the sliding mode observer. However, in order to achieve the better control effect in practice engineering, it is generally difficult to obtain the exact information of the size of system fault from an FDI strategy because of the existence of the unexpected model uncertainties, disturbances, perturbations, and noises that may also occur in the fault systems [16]. For increased efficiency, the problem of fault estimation (FE) has stirred renewed research interest; accordingly, a variety of important results have been developed in the literatures [17-23]. Compared with FDI, FE can give the size and shape of the fault and can thus automatically perform the required fault detection. However, FE is a more challenging task because it requires an estimation of the location after the alarm has been set, and the size of the fault should be made.

Recently, many fault estimation approaches based on robust or adaptive observers have been developed in the literature. The problem of fault estimation and fault-tolerant control for stochastic systems and nonlinear descriptor systems were studied in $[24,25]$. Also, Liu et al. [26] investigate the fault-tolerant control for nonlinear Markovian jump systems based on the sliding mode observer technique. In [27], the problem of fault estimation for a class of linear parameter varying Markovian jump systems with the actuator and sensor faults was studied based on the Cholesky decomposition technique. Based on the Lyapunov-Razumikhin method, an iterative algorithm is proposed to solve the robust fault estimation for a class of uncertain networked control systems in [28]. The literature [29] presents a novel method to address a proportional integral observer design for the actuator and sensor faults' estimation based on the T-S fuzzy model with unmeasurable premise variables. Based on a discrete mean-value convergence lemma, an improved iterative observer for fault estimation of a class of discrete-time T-S fuzzy systems is discussed in [30]. By introducing the reset element in the observer, Pourdehi and Karimaghaee [31] investigated the problem of actuator fault estimation and FTC for a class of T-S fuzzy nonlinear timedelay systems. You et al. [19] proposed a proportional multiple integral observer to achieve simultaneous estimation of system states, time-varying actuator, and sensor faults for T-S fuzzy systems.

It is well known that the presence of time delay often degrades system performances and may even lead to instability; many criteria have been advanced for checking the stability of MJSs. Because the delay-dependent results usually have less conservatism in contrast to the delay-independent ones, hence, some improved approaches have been proposed for deriving the delay-dependent results on linear MJSs with time delays, such as delay decomposition method [32, 33] and free weighting method [34, 35]. Based on the abovementioned methods, the delay-dependent fault estimation and parity space-based fault estimation schemes are studied in $[36,37]$. The result in [17] is developed to research the fault estimation problem based on the condition that the time delay is constant, but the external disturbance is not considered, which inevitably brings some conservativeness. Chen et al. [18] investigate the problem of FE for a class of Markovian jump systems with time-varying delay, but the delay mode is the same as that for jump in other system states. In [38], robust $H_{\infty}$ control for a class of uncertain stochastic Markovian jump systems with interval and distributed time-varying delays is researched, but the problem of FE is not considered. It should be mentioned that although there are many results regarding the fault estimation for time delay T-S fuzzy jump systems, all such works have considered only time delay that share the same mode with system matrices, and the time delay have a fixed type under different fuzzy rules. Different from the abovementioned delays, in practical systems, with the change of time $t$, the state delays respond to changes in system modes. Therefore, in order to ensure a stable and efficient work system, the mode-dependent time-varying state delay is an important subject to be researched. In summary, despite these efforts to deal with time delay MJSs, to the best of our knowledge, there are only few conclusions of robust fault estimation for T-S fuzzy MJSs with actuator fault, external distu rbance, and mode-dependent interval time-varying delay, simultaneously, which has not been fully investigated. This motivates the present study.

In this paper, we are concerned with the problem of robust fault estimation for a class of T-S fuzzy MJSs with external disturbances and mode-dependent time-delay parameters. Our aim is to design a mode-dependent fuzzy FE observer such that the error dynamic is stochastically stable with a prescribed $H_{\infty}$ performance of disturbance attenuation. Meanwhile, the proposed adaptive fault estimation algorithm can enhance the rapidity and accuracy performance of fault estimation. The main contributions of this paper are outlined as follows:

(i) Different from the existing results in $[17,20,22]$, the mode-dependent time-varying delay is considered, which may not be the same as that for jump in system states. Then, a fast fault estimation observer is proposed, which can not only provide estimations of the actuator fault but also guarantee the good performance when the system and time delay change in different modes.

(ii) Both lower-upper bounds and the integral term of mode-dependent delays are considered in the construction of the LKF function. Moreover, a derivative term is added on the basis of fault estimation equation in the estimation algorithm. The improved modedependent fault estimator existence criteria are obtained than existing results in $[17,18,22]$.

(iii) The robust fault estimation criterion of the error dynamic system can be readily obtained by means of LMIs. Compared with the existing results in [22], the 
proposed method has the advantage of few variables and wider application. Three numerical examples are provided to show the effectiveness of the proposed method.

The rest of this paper is organized as follows. The system description and problem formulations are presented in Section 2. Section 3 presents the main results on the robust FE observer design scheme. In Section 4, numerical examples are presented to demonstrate the effectiveness of the proposed methods. Finally, Section 5 concludes the paper.

Notations: throughout the paper, $\mathbb{R}^{n}$ denotes the $n$-dimensional real Euclidean space, $I$ denotes the identity matrix, the superscripts $T$ and -1 stand for the matrix transpose and inverse, respectively, notation $X>0(X \geq 0)$ means that matrix $X$ is real symmetric positive definite (positive semidefinite), and $\|\cdot\|$ is the spectral norm. The symbol / * " stands for matrix block induced by symmetry. If not explicitly stated, all matrices are assumed to have compatible dimensions for algebraic operations.

\section{Problem Formulation}

Let us consider a class of nonlinear MJSs with mode-dependent time-varying state delays described by the following T-S fuzzy models over the probability space $\left(\Omega, M, P_{r}\right)$, where $\Omega$ is the sample space, $M$ is the algebra of events, and $P_{r}$ is the probability measure defined on $M$. THEN

$$
\left\{\begin{array}{l}
\dot{x}(t)=A_{i}\left(r_{t}\right) x(t)+A_{d i}\left(r_{t}\right) x\left(t-\mathrm{d}\left(t, l_{t}\right)\right)+B_{i}\left(r_{t}\right) u(t)+B_{f i}\left(r_{t}\right) f(t)+D_{i}\left(r_{t}\right) \omega(t) \\
y(t)=C_{i}\left(r_{t}\right) x(t) \\
x(t)=\alpha(t), \quad r_{t}=\beta(t), l_{t}=\gamma(t), \forall t \in\left[-h_{2}, 0\right], i=1,2, \ldots, s
\end{array}\right.
$$

where $x(t) \in \mathbb{R}^{n}$ is the state of the nonlinear model, $y(t) \in \mathbb{R}^{l}$ stands for the measured output, $u(t) \in \mathbb{R}^{w}$ denotes the controlled input, $\omega(t) \in \mathbb{R}^{m}$ is the exogenous disturbance that belongs to $L_{2}[0, \infty), f(t) \in \mathbb{R}^{q}$ represents the possible actuator fault, $M_{i j}(i=1,2, \ldots, s, j=1,2, \ldots, p)$ are fuzzy sets, $\xi(t)=\left[\xi_{1}(t), \ldots, \xi_{p}(t)\right]^{T}$ is the available premise variable vector that does not depend on the input variables $u(t)$, $A_{i}\left(r_{t}\right), A_{d i}\left(r_{t}\right), B_{i}\left(r_{t}\right), B_{f i}\left(r_{t}\right), C_{i}\left(r_{t}\right)$, and $D_{i}\left(r_{t}\right)$ are known mode-dependent constant matrices with appropriate dimensions, and $\mathrm{d}\left(t, l_{t}\right)$ is the mode-dependent time-varying delay satisfying $0 \leq h_{1 l} \leq \mathrm{d}\left(t, l_{t}\right) \leq h_{2 l}<\infty \quad$ and $\dot{\mathrm{d}}\left(t, l_{t}\right) \leq h_{3 l}<1$.

Here, let the random process $\left\{r_{t}, t \geq 0\right\}$ and $\left\{l_{t}, t \geq 0\right\}$ be continuous-time discrete-state Markov stochastic processes taking values on a finite set $\Lambda_{1}=\left\{1,2, \ldots, N_{1}\right\}$ and $\Lambda_{2}=$ $\left\{1,2, \ldots, N_{2}\right\}$ with transition rate matrix $\Pi_{1}=\left\{\pi_{r k}\right\}$, $r, k \in \Lambda_{1}$, and $\Pi_{2}=\left\{\pi_{l m}\right\}, l, m \in \Lambda_{2}$, respectively. Meanwhile, we also assume that $\left\{r_{t}, t \geq 0\right\}$ and $\left\{l_{t}, t \geq 0\right\}$ are mutually independent. The transition probabilities from mode $r$ at time $t$ to mode $k$ at time $t+\Delta t$ of the random Markov process $\left\{r_{t}, t \geq 0\right\}$ and from mode $l$ at time $t$ to mode $m$ at time $t+\Delta t$ of the random Markov process $\left\{l_{t}, t \geq 0\right\}$ are

$$
\begin{gathered}
P_{r}=P_{r}\left\{r_{t+\Delta t}=k \mid r_{t}=r\right\}= \begin{cases}\pi_{r k} \Delta t+o(\Delta t), & \text { if } r \neq k, \\
1+\pi_{r r} \Delta t+o(\Delta t), & \text { if } r=k,\end{cases} \\
P_{l}=P_{l}\left\{l_{t+\Delta t}=m \mid l_{t}=l\right\}= \begin{cases}\pi_{l m} \Delta t+o(\Delta t), & \text { if } l \neq m, \\
1+\pi_{l l} \Delta t+o(\Delta t), & \text { if } l=m,\end{cases}
\end{gathered}
$$

where $\Delta t>0$ and $((o(\Delta t)) / \Delta t) \longrightarrow 0$ when $\Delta t \longrightarrow 0 . \pi_{r k} \geq 0$ and $\pi_{l m} \geq 0$ are, respectively, the transition probability rates from mode $r$ at time $t$ to mode $k$ and from mode $l$ to mode $m$, with $\sum_{k=1, k \neq r}^{N_{1}} \pi_{r k}=-\pi_{r r}$ and $\sum_{m=1, m \neq l}^{N_{2}} \pi_{l m}=-\pi_{l l} \cdot \alpha(t)$ is the vector-valued initial continuous function defined on a interval $\left[-h_{2}, 0\right]$, where $h_{2}=\max \left\{h_{2 l}, l \in \Lambda_{2}\right\}$, and $\beta(t)$ and $\gamma(t)$ are, respectively, the initial mode.

For convenience, we denote $A_{i}\left(r_{t}\right), A_{d i}\left(r_{t}\right), B_{i}\left(r_{t}\right)$, $B_{f i}\left(r_{t}\right), C_{i}\left(r_{t}\right), D_{i}\left(r_{t}\right)$, and $x\left(t-\mathrm{d}\left(t, l_{t}\right)\right)$ as $A_{i}(r), A_{d i}(r)$, $B_{i}(r), B_{f i}(r), C_{i}(r), D_{i}(r)$, and $x(t-\mathrm{d}(l))$, respectively. Before proceeding further, we will introduce some definitions and assumptions to be needed in the development of main results through this paper.
Definition 1. The T-S fuzzy MJSs (when $u(t)=f(t)=\omega(t)=0)$ are said to be stochastically stable if, for any initial $x(t)=\alpha(t)$ and initial mode $r_{t}=\beta(t), l_{t}=$ $\gamma(t)$ has

$$
\lim _{T \rightarrow \infty} E\left\{\int_{0}^{T}\|x[t, \alpha(t), \beta(t), \gamma(t)]\|^{2} \mathrm{~d} t \mid x(t)=\alpha(t), \beta(t), \gamma(t)\right\}<\infty .
$$

Definition 2. In the Euclidean space $\left\{\mathbb{R}^{n} \times \Lambda_{1} \times \Lambda_{2} \times \mathbb{R}_{+}\right\}$, we introduce a stochastic positive functional as $V\left[x(t), r_{t}=r, l_{t}=l, t>0\right]$, the weak infinitesimal operator of which satisfies 


$$
\begin{aligned}
\Im V[x(t), r, l, t]= & \lim _{\Delta t \longrightarrow 0} \frac{1}{\Delta t}\left[E\left\{V\left(x(t+\Delta t), r_{t+\Delta t}, l_{t+\Delta t}\right)\right\}\right. \\
& -V(x(t), r, l, t)] .
\end{aligned}
$$

Assumption 1.

$$
\operatorname{Rank}\left(C_{i}(r) B_{f i}(r)\right)=q
$$

Assumption 2. Invariant zeros of $\left(A_{i}(r) B_{f i}(r) C_{i}(r)\right)$ lie in open left-half plane.

Remark 1. Assumptions 1 and 2 mean that the triple $\left(A_{i}(r) B_{f i}(r) C_{i}(r)\right)$ has relative degree one and is minimumphase. Generally speaking, Assumptions 1 and 2 hold if and only if symmetric positive definite matrices $P(r, l), Q$ and matrices $L_{i}(r, l), H_{i}(r, l)$ exist, satisfying the following conditions:

$$
\begin{aligned}
& P(r, l)\left(A_{i}(r)-L_{i}(r, l) C_{i}(r)\right)+\left(A_{i}(r)-L_{i}(r, l) C_{i}(r)\right)^{T} \\
& P(r, l)=-Q, \\
& B_{f i}^{T}(r) P(r, l)=H_{i}(r, l) C_{i}(r) .
\end{aligned}
$$

Therefore, Assumption 1 usually holds for the cases where the number of output is greater than or equal to the number of fault inputs; Assumption 2 is satisfied for most systems [20].

Remark 2. The external disturbance input $\omega(t)$ is arbitrary energy bounded in the considered system (1), and then, it is not necessary to obtain its exact knowledge such as the statistics. Moreover, the time-varying delay $\mathrm{d}\left(t, l_{t}\right)$ is modedependent differentiable and the delay mode $l_{t}$ is not the same as that for jump in the system state mode $r_{t}$. Therefore, the system state delay is the less conservative form and the application scope of the system mode is wide.

Then, by using a standard fuzzy singleton inference method, i.e., a singleton fuzzifier to produce a fuzzy inference and weighted center-average defuzzifer, the T-S fuzzy dynamic MJSs can be obtained as follows:

$$
\left\{\begin{array}{l}
\dot{x}(t)=\sum_{i=1}^{s} \mu_{i}(t)\left\{A_{i}(r) x(t)+A_{d i}(r) x(t-d(l))+B_{i}(r) u(t)+B_{f i}(r) f(t)+D_{i}(r) \omega(t)\right\} \\
y(t)=\sum_{i=1}^{s} \mu_{i}(t) C_{i}(r) x(t) \\
x(t)=\alpha(t), \quad r_{t}=\beta(t), l_{t}=\gamma(t), \forall t \in\left[-h_{2}, 0\right]
\end{array}\right.
$$

in which $\mu_{i}(t)=\mu_{i}(\xi(t))=\left(\beta_{i}(\xi(t)) / \sum_{i=1}^{s} \beta_{i}(\xi(t))\right)$, where $\beta_{i}(\xi(t))=\prod_{i=1}^{p} M_{i j}\left(\xi_{j}(t)\right)$, and $M_{i j}\left(\xi_{j}(t)\right)$ represents the grade of membership of $\xi_{j}(t)$ in $M_{i j}$. Here, we always assume that $\beta_{i}(\xi(t)) \geq 0, i=1, \ldots, s, \sum_{i=1}^{s} \beta_{i}(\xi(t))>0$ for any $\xi(t)$. Hence, $\quad \mu_{i}(\xi(t))$ satisfy $\mu_{i}(\xi(t)) \geq 0, i=1, \ldots, s, \sum_{i=1}^{s} \mu_{i}(\xi(t))=1$ for any $\xi(t)$. In order to estimate the fault, our task is to design a suitable fault estimation observer. First, we consider the following T$S$ fuzzy dynamic jumping observer. THEN
Observer rule $i$ : IF $\xi_{1}(t)$ is $M_{i 1}$, and $\ldots$, and $\xi_{p}(t)$ is $M_{i p}$,

$$
\left\{\begin{array}{l}
\dot{\hat{x}}(t)=A_{i}(r) \hat{x}(t)+A_{d i}(r) \widehat{x}(t-\mathrm{d}(l))+B_{i}(r) u(t)+B_{f i}(r) \hat{f}(t)+L_{i}(r, l)(y(t)-\hat{y}(t))+L_{d i}(r, l)\left(y_{d}(t)-\hat{y}_{d}(t)\right), \\
\hat{y}(t)=C_{i}(r) \widehat{x}(t), \\
\hat{x}(t)=\bar{\alpha}(t), \quad \forall t \in\left[-h_{2}, 0\right],
\end{array}\right.
$$

and the global T-S fuzzy dynamic jumping observer model can be constructed as 


$$
\left\{\begin{array}{l}
\dot{\hat{x}}(t)=\sum_{i=1}^{s} \mu_{i}(t)\left\{\begin{array}{c}
A_{i}(r) \hat{x}(t)+A_{d i}(r) \widehat{x}(t-\mathrm{d}(l))+B_{i}(r) u(t)+L_{i}(r, l)(y(t)-\hat{y}(t)) \\
+B_{f i}(r) \widehat{f}(t)+L_{d i}(r, l)\left(y_{d}(t)-\widehat{y}_{d}(t)\right)
\end{array}\right\}, \\
\hat{y}(t)=\sum_{i=1}^{s} \mu_{i}(t) C_{i}(r) \widehat{x}(t), \\
\hat{x}(t)=\bar{\alpha}(t), \quad \forall t \in\left[-h_{2}, 0\right],
\end{array}\right.
$$

where $\hat{x}(t)$ is the observer state, $\hat{y}(t)$ is the observer output, and $\bar{\alpha}(t)$ is the initial estimation state. Here, we assume that the system matrices and mode-dependent time-varying delay of the observer have the same process as system (7). So, the initial mode value is defined as $r_{t}=\beta(t)$ and $l_{t}=\gamma(t)$, where $\beta(t)$ and $\gamma(t)$ are, respectively, the initial mode in system (7). This assumption for fuzzy jumping systems is common in the existing literature $[11,17,18,39]$. Denote the state and fault estimation errors as $e_{x}(t)=x(t)-\hat{x}(t)$ and $e_{f}(t)=f(t)-\widehat{f}(t)$, and we can obtain the following error dynamic system by combining (7) and (9):

$$
\left\{\begin{array}{l}
\dot{e}_{x}(t)=\sum_{i=1}^{s} \sum_{j=1}^{s} \mu_{i}(\xi(t)) \mu_{j}(\xi(t))\left\{\begin{array}{c}
{\left[A_{i}(r)-L_{i}(r) C_{j}(r)\right] e_{x}(t)+B_{f i}(r) e_{f}(t)} \\
+\left[A_{d i}(r)-L_{d i}(r) C_{j}(r)\right] e_{x}(t-d(l))+D_{i}(r) \omega(t)
\end{array}\right\} \\
=\widehat{A}(r) e_{x}(t)+\widehat{A}_{d}(r) e_{x}(t-\mathrm{d}(l))+\widehat{B}_{f}(r) e_{f}(t)+\widehat{D}(r) \omega(t), \\
e_{y}(t)=\sum_{i=1}^{s} \mu_{i}(\xi(t)) C_{i}(r) e_{x}(t)=\widehat{C}(r) e_{x}(t) .
\end{array}\right.
$$

By comparison with the conventional adaptive fault estimation algorithm, we consider the following fast adaptive fault estimation algorithm for fuzzy Markovian jumping systems with mode-dependent time-varying state delays. In our algorithm, we add $e_{y}(t)$ in the estimation equation, that is,

$$
\begin{aligned}
\widehat{f}(t)= & -\Gamma \sum_{i=1}^{s} \mu_{i}(\xi(t)) H_{i}(r, l)\left[\int_{t_{f}}^{t} e_{y}(s) \mathrm{d} s+e_{y}(t)\right] \\
& =-\Gamma \widehat{H}(r, l)\left[\int_{t_{f}}^{t} e_{y}(s) \mathrm{d} s+e_{y}(t)\right],
\end{aligned}
$$

where the matrix $\Gamma$ is the learning rate and $t_{f}$ denotes the instant when fault occurs. When $\omega(t) \in L_{2}[0, \infty)$ and $f(t) \neq 0$, in order to minimize the external disturbance effect on fault estimation, for $T>0$, we introduce the following robust $H_{\infty}$ performance index:

$$
J(T)=E\left\{\int_{0}^{T}\left[z^{T}(t) z(t)-\gamma^{2} \omega^{T}(t) \omega(t)\right] \mathrm{d} t\right\},
$$

where $\gamma>0$ is a given scalar reflecting disturbance attenuation level and the signal $z(t)=L^{\prime} e_{x}(t)$ is a weighted estimation error. Then, the objective of robust $H_{\infty}$ fault estimation is to design observer (9) such that the error system (10) has a $H_{\infty}$ performance $\gamma$ for all admissible disturbance, i.e., the system satisfies the following: (i) the error system (10) with $\omega(t)=0$ is stochastically stable and (ii) the $H_{\infty}$ performance (12) is guaranteed for all nonzero $\omega(t) \in L_{2}[0, \infty)$ and the fault estimation algorithm can realize that $\lim _{t \rightarrow \infty} e_{f}(t)=0$.

\section{Main Results}

In this section, the robust FE observer design method will be proposed not only to evidently improve performances of fault estimation but also to guarantee the filtering error system to be stochastically stable with a prescribed $H_{\infty}$ performance. The main results are presented in the following theorem.

Theorem 1. For the given scalars $\gamma>0$ and $0<h_{1}<h_{2}$, the error dynamic system (10) with mode-dependent time-varying delays is stochastically stable (when $\omega(t)=f(t)=0$ ) and has a prescribed $H_{\infty}$ performance level $\gamma\left(\right.$ when $\omega(t) \in L_{2}[0, \infty)$ ) if there exist mode-dependent symmetric positive-definite matrix $P(r, l)>0, Q>0, Z_{i}>0(i=1,2), W>0, G>0$, $\widehat{H}(r, l), \quad$ and $\quad N=\left[\begin{array}{lllll}N_{1}^{T} & N_{2}^{T} & N_{3}^{T} & 0 & N_{4}^{T}\end{array}\right]$ and $M=\left[\begin{array}{lllll}M_{1}^{T} & M_{2}^{T} & M_{3}^{T} & 0 & M_{4}^{T}\end{array}\right]$, such that the following matrix inequalities hold for all $r, k \in \Lambda_{1}, l, m \in \Lambda_{2}$ : 


$$
\begin{aligned}
& \widehat{H}(r, l) \widehat{C}(r)=\widehat{B}_{f}^{T}(r) P(r, l), \\
& {\left[\begin{array}{ccccc}
\Xi(r, l) & h_{2} M^{T} & h_{12} N^{T} & h_{2} \Gamma^{T}(r) P(r, l) & h_{12} \Gamma^{T}(r) P(r, l) \\
* & -h_{2} Z_{1} & 0 & 0 & 0 \\
* & * & -h_{12} Z_{2} & 0 & 0 \\
* & * & * & -h_{2} P(r, l) Z_{1}^{-1} P(r, l) & 0 \\
* & * & * & * & -h_{12} P(r, l) Z_{2}^{-1} P(r, l)
\end{array}\right]<0}
\end{aligned}
$$

where $h_{1}=\min \left\{h_{1 l}, l \in \Lambda_{2}\right\}, h_{2}=\max \left\{h_{2 l}, l \in \Lambda_{2}\right\}$,

$$
\begin{aligned}
& \Xi(r, l)=\left[\begin{array}{ccccc}
\Xi_{11} & \Xi_{12} & \Xi_{13} & \Xi_{14} & \Xi_{15} \\
* & \Xi_{22} & \Xi_{23} & \Xi_{24} & \Xi_{25} \\
* & * & \Xi_{33} & 0 & -N_{4}^{T} \\
* & * & * & \Xi_{44} & \Xi_{45} \\
* & * & * & * & -\gamma^{2} I
\end{array}\right] \\
& \Gamma^{T}(r)=\left[\begin{array}{c}
\widehat{A}^{T}(r) \\
\widehat{A}_{d}^{T}(r) \\
0 \\
\widehat{B}_{f}^{T}(r) \\
\widehat{D}^{T}(r)
\end{array}\right] \text {, } \\
& \Xi_{11}=P(r, l) \widehat{A}(r)+\widehat{A}^{T}(r) P(r, l)+\sum_{k=1}^{N_{1}} \pi_{r k} P(k, l)+\sum_{m=1}^{N_{2}} \pi_{l m} P(r, m) \\
& +\left(1+\pi h_{12}\right) W+Q+L \prime^{T} L^{\prime}+M_{1}+M_{1}^{T}, \quad \pi=\max \left\{\pi_{r r}, r \in \Lambda_{1}\right\}, \\
& \Xi_{12}=P(r, l) \widehat{A}_{d}(r)-M_{1}+M_{2}^{T}+N_{1} \text {, } \\
& \Xi_{13}=M_{3}^{T}-N_{1} \text {, } \\
& \Xi_{14}=-\widehat{A}^{T}(r) P(r, l) \widehat{B}_{f}(r), \\
& \Xi_{15}=P(r, l) \widehat{D}(r)+M_{4}^{T}, \\
& \Xi_{22}=-\left(1-h_{3 l}\right) W-M_{2}-M_{2}^{T}+N_{2}+N_{2}^{T} \text {, } \\
& \Xi_{23}=-M_{3}^{T}+N_{3}^{T}-N_{2} \text {, } \\
& \Xi_{24}=-\widehat{A}_{d}(r) P(r, l) \widehat{B}_{f}(r), \\
& \Xi_{25}=-M_{4}^{T}+N_{4}^{T} \text {, } \\
& \Xi_{33}=-Q-N_{3}-N_{3}^{T}, \\
& \Xi_{44}=G-2 \widehat{B}_{f}(r) P(r, l) \widehat{B}_{f}(r), \\
& \Xi_{45}=-\widehat{B}_{f}^{T}(r) P(r, l) \widehat{D}(r) .
\end{aligned}
$$


Proof. The process of proof can be classified into three parts.

Part 1. Construct the Lyapunov-Krasovskii function.

Part 2. Make the error dynamic (10) stochastically stable when $\omega(t)=0$ and $f(t)=0$.

Part 3. Find the observer gains $L_{i}(r, l)$ and $L_{d i}(r, l)$ to make that the $H_{\infty}$ norm of dynamic (10) is less than the prescribed value $\gamma$ when the exogenous disturbance signal $\omega(t) \neq 0$. At the same time, the fault is estimated by algorithm (11).

Part 1. For the error dynamic (10), we define the stochastic Lyapunov-Krasovskii functional as $V\left[e_{x}(t), r, l, t\right]$ $=V_{1}\left[e_{x}(t), r, l, t\right] \quad+V_{2}\left[e_{x}(t), r, l, t\right]+V_{3}\left[e_{x}(t), r, l\right.$, $t]+V_{4}\left[e_{x}(t), r, l, t\right]$, where

$$
\begin{aligned}
& V_{1}\left[e_{x}(t), r, l, t\right]=e_{x}^{T}(t) P(r, l) e_{x}(t), \\
& V_{2}\left[e_{x}(t), r, l, t\right]=\int_{t-h_{2}}^{t} e_{x}^{T}(s) Q e_{x}(s) \mathrm{d} s+\int_{t-d\left(t, l_{t}\right)}^{t} e_{x}^{T}(s) W e_{x}(s) \mathrm{d} s, \\
& V_{3}\left[e_{x}(t), r, l, t\right]=\pi \int_{-h_{2}}^{-h_{1}} \int_{t+\theta}^{t} e_{x}^{T}(s) W e_{x}(s) \mathrm{d} s \mathrm{~d} \theta+\int_{-h_{2}}^{0} \int_{t+\theta}^{t} \dot{e}_{x}^{T}(s) Z_{1} \dot{e}_{x}(s) \mathrm{d} s \mathrm{~d} \theta+\int_{-h_{2}}^{-h_{1}} \int_{t+\theta}^{t} \dot{e}_{x}^{T}(s) Z_{2} \dot{e}_{x}(s) \mathrm{d} s \mathrm{~d} \theta, \\
& V_{4}\left[e_{x}(t), r, l, t\right]=e_{f}^{T}(t) \Gamma^{-1} e_{f}(t) .
\end{aligned}
$$

By recalling the weak infinitesimal operator $\mathfrak{I}$ in Definition 2, the time derivative of $V\left[e_{x}(t), r, l, t\right]$ along the trajectories of the overall global T-S fuzzy error dynamic systems (10) is given as

$$
\begin{aligned}
& \mathfrak{J} V_{1}\left[e_{x}(t), r, l, t\right]=\lim _{\Delta t \rightarrow 0} \frac{1}{\Delta t} \mathbf{E}\left\{e_{x}^{T}(t+\Delta t) P\left(r_{t+\Delta t}, l_{t+\Delta t}\right) e_{x}(t+\Delta t)-e_{x}^{T}(t) P\left(r_{t}, l_{t}\right) e_{x}(t)\right\} \\
& =\lim _{\Delta t \rightarrow 0} \frac{1}{\Delta t}\left\{e_{x}^{T}(t+\Delta t)\left[1+\pi_{r r} \Delta t+o(\Delta t)\right]\left[1+\pi_{l l} \Delta t+o(\Delta t)\right] P\left(r_{t}, l_{t}\right) e_{x}(t+\Delta t)\right. \\
& -e_{x}^{T}(t+\Delta t)\left[1+\pi_{r r} \Delta t+o(\Delta t)\right]\left(\sum_{m=1}^{N_{2}}\left[1+\pi_{l m} \Delta t+o(\Delta t)\right]\right) P\left(r_{t}, m_{t}\right) e_{x}(t+\Delta t) \\
& +e_{x}^{T}(t+\Delta t)\left[1+\pi_{l l} \Delta t+o(\Delta t)\right]\left(\sum_{k=1}^{N_{1}}\left[1+\pi_{r k} \Delta t+o(\Delta t)\right]\right) P\left(k_{t}, l_{t}\right) e_{x}(t+\Delta t) \\
& \left.+e_{x}^{T}(t+\Delta t)\left(\sum_{m=1}^{N_{2}}\left[1+\pi_{l m} \Delta t+o(\Delta t)\right]\right)\left(\sum_{k=1}^{N_{1}}\left[1+\pi_{r k} \Delta t+o(\Delta t)\right]\right) \times P\left(k_{t}, m_{t}\right) e_{x}(t+\Delta t)-e_{x}^{T}(t) P\left(k_{t}, l_{t}\right) e_{x}(t)\right) \\
& =2 e_{x}^{T}(t) P\left(r_{t}, l_{t}\right)\left(\widehat{A}(r) e_{x}(t)+\widehat{A}_{d}(r) e_{x}(t-d(l))+\widehat{B}_{f}(r) e_{f}(t)+\widehat{D}(r) \omega(t)\right) \\
& +e_{x}^{T}(t)\left(\sum_{m=1}^{N_{2}} \pi_{l m} P\left(r_{t}, m_{t}\right)+\sum_{k=1}^{N_{1}} \pi_{r k} P\left(k_{t}, l_{t}\right)\right) e_{x}(t) \\
& \mathfrak{I} V_{2}\left[e_{x}(t), r, l, t\right]=e_{x}^{T}(t) Q e_{x}(t)-e_{x}^{T}\left(t-h_{2}\right) Q e_{x}\left(t-h_{2}\right)+\lim _{\Delta t \rightarrow 0} \frac{1}{\Delta t} \mathbf{E}\left\{\int_{t+\Delta t-d\left(t+\Delta t, l_{t+\Delta t}\right)}^{t+\Delta t} e_{x}^{T}(s) W e_{x}(s) \mathrm{d} s-\int_{t-d\left(t, l_{t}\right)}^{t} e_{x}^{T}(s) W e_{x}(s) \mathrm{d} s\right\}, \\
& =e_{x}^{T}(t) Q e_{x}(t)-e_{x}^{T}\left(t-h_{2}\right) Q e_{x}\left(t-h_{2}\right)+\sum_{m=1}^{N_{2}} \pi_{l m} \int_{t-d(m)}^{t} e_{x}^{T}(s) W e_{x}(s) \mathrm{d} s \\
& +\lim _{\Delta t \rightarrow 0} \frac{1}{\Delta t}\left(\int_{t+\Delta t-d\left(t, l_{t}\right)}^{t-d\left(t, l_{t}\right)} e_{x}^{T}(s) W e_{x}(s) \mathrm{d} s+\int_{t}^{t+\Delta t} e_{x}^{T}(s) W e_{x}(s) \mathrm{d} s\right) \\
& =e_{x}^{T}(t)(Q+W) e_{x}(t)-e_{x}^{T}\left(t-h_{2}\right) Q e_{x}\left(t-h_{2}\right)-(1-\dot{d}(l)) e_{x}^{T}(t-d(l)) W e_{x}(t-d(l))+\sum_{m=1}^{N_{2}} \pi_{l m} \int_{t-d(m)}^{t} e_{x}^{T}(s) W e_{x}(s) \mathrm{d} s .
\end{aligned}
$$


Noticing that $\pi_{l m} \geq 0$ for $l \neq m$ and $\pi_{l l} \leq 0$, we can get that

$$
\begin{aligned}
\sum_{m=1}^{N_{2}} \pi_{l m} \int_{t-d(m)}^{t} e_{x}^{T}(s) W e_{x}(s) \mathrm{d} s & =\sum_{l \neq m} \pi_{l m} \int_{t-d(m)}^{t} e_{x}^{T}(s) W e_{x}(s) \mathrm{d} s+\pi_{l l} \int_{t-d(m)}^{t} e_{x}^{T}(s) W e_{x}(s) \mathrm{d} s \\
& \leq \sum_{l \neq m} \pi_{l m} \int_{t-h_{2}}^{t} e_{x}^{T}(s) W e_{x}(s) \mathrm{d} s+\pi_{l l} \int_{t-h_{1}}^{t} e_{x}^{T}(s) W e_{x}(s) \mathrm{d} s \\
& =-\pi_{l l} \int_{t-h_{2}}^{t} e_{x}^{T}(s) W e_{x}(s) \mathrm{d} s+\pi_{l l} \int_{t-h_{1}}^{t} e_{x}^{T}(s) W e_{x}(s) \mathrm{d} s \\
& \leq \pi \int_{t-h_{2}}^{t-h_{1}} e_{x}^{T}(s) W e_{x}(s) \mathrm{d} s .
\end{aligned}
$$

Then, we denote $h_{12}=h_{2}-h_{1}$, and the time derivative of $V_{3}\left[e_{x}(t), r, l, t\right]$ is as follows:

$$
\begin{aligned}
\Im V_{3}\left[e_{x}(t), r, l, t\right]= & \pi h_{12} e_{x}^{T}(t) W e_{x}(t)-\pi \int_{t-h_{2}}^{t-h_{1}} e_{x}^{T}(s) W e_{x}(s) \mathrm{d} s+\dot{e}_{x}^{T}(t)\left(h_{2} Z_{1}+h_{12} Z_{2}\right) \dot{e}_{x}(t) \\
& -\int_{t-h_{2}}^{t} \dot{e}_{x}^{T}(s) Z_{1} \dot{e}_{x}(s) \mathrm{d} s-\int_{t-h_{2}}^{t-h_{1}} \dot{e}_{x}^{T}(s) Z_{2} \dot{e}_{x}(s) \mathrm{d} s \leq \pi h_{12} e_{x}^{T}(t) W e_{x}(t)-\pi \int_{t-h_{2}}^{t-h_{1}} e_{x}^{T}(s) W e_{x}(s) \mathrm{d} s \\
& +\dot{e}_{x}^{T}(t)\left(h_{2} Z_{1}+h_{12} Z_{2}\right) \dot{e}_{x}(t)-\int_{t-d(l)}^{t} \dot{e}_{x}^{T}(s) Z_{1} \dot{e}_{x}(s) \mathrm{d} s-\int_{t-h_{2}}^{t-d(l)} \dot{e}_{x}^{T}(s) Z_{2} \dot{e}_{x}(s) \mathrm{d} s .
\end{aligned}
$$

Based on the assumption that the derivative of $f(t)$ with respect to time is norm-bounded $\|\dot{f}(t)\| \leq f_{1}$, it is easy to show that, for a symmetric positive definite matrix $G$, it can obtain that

$$
\begin{aligned}
2 e_{f}^{T}(t) \Gamma^{-1} \dot{f}(t) & \leq e_{f}^{T}(t) G e_{f}(t)+\dot{f}^{T}(t) \Gamma^{-1} G^{-1} \Gamma^{-1} \dot{f}(t) \\
& \leq e_{f}^{T}(t) G e_{f}(t)+\delta
\end{aligned}
$$

where $\delta=f_{1}^{2} \cdot \lambda_{\max }\left(\Gamma^{-1} G^{-1} \Gamma^{-1}\right)$. So, it can get the derivative of $V_{4}\left[e_{x}(t), r, l, t\right]$ as

$$
\begin{aligned}
\mathfrak{J} V_{4}\left[e_{x}(t), r, l, t\right]= & -2 e_{f}^{T}(t) H(r, l) \widehat{C}(r)\left(\dot{e}_{x}(t)+e_{x}(t)\right)+2 e_{f}^{T}(t) \Gamma^{-1} \dot{f}(t) \\
= & -2 e_{f}^{T}(t) \widehat{B}_{f}^{T}(r) P(r, l) \widehat{A}(r) e_{x}(t)-2 e_{f}^{T}(t) \widehat{B}_{f}^{T}(r) P(r, l) \widehat{A}_{d}(r) e_{x}(t-\mathrm{d}(l))-2 e_{f}^{T}(t) \widehat{B}_{f}^{T}(r) P(r, l) \widehat{B}_{f}(r) e_{f}(t) \\
& -2 e_{f}^{T}(t) \widehat{B}_{f}^{T}(r) P(r, l) \widehat{D}(r) \omega(t)-2 e_{f}^{T}(t) \widehat{B}_{f}^{T}(r) P(r, l) e_{x}(t)+e_{f}^{T}(t) G e_{f}(t)+\delta .
\end{aligned}
$$

Part 2. Taking into account $\omega(t)=0$ and $f(t)=0$, the weak infinitesimal operator $\mathfrak{\Im}$ of $V\left[e_{x}(t), r, l, t\right]$ can be obtained:

$$
\begin{aligned}
\mathfrak{S} V\left[e_{x}(t), r, l, t\right]= & 2 e_{x}^{T}(t) P\left(r_{t}, l_{t}\right) \dot{e}_{x}(t)+e_{x}^{T}(t)\left(\sum_{m=1}^{N_{2}} \pi_{l m} P\left(r_{t}, m_{t}\right)+\sum_{k=1}^{N_{1}} \pi_{r k} P\left(k_{t}, l_{t}\right)\right) e_{x}(t) \\
& +e_{x}^{T}(t)(Q+W) e_{x}(t)-e_{x}^{T}\left(t-h_{2}\right) Q e_{x}\left(t-h_{2}\right)-(1-\dot{d}(l)) e_{x}^{T}(t-d(l)) W e_{x}(t-d(l)) \\
& +\sum_{m=1}^{N_{2}} \pi_{l m} \int_{t-d(m)}^{t} e_{x}^{T}(s) W e_{x}(s) \mathrm{d} s+\pi h_{12} e_{x}^{T}(t) W e_{x}(t)-\pi \int_{t-h_{2}}^{t-h_{1}} e_{x}^{T}(s) W e_{x}(s) \mathrm{d} s+h_{2} \dot{e}_{x}^{T}(t) Z_{1} \dot{e}_{x}(t) \\
& -\int_{t-h_{2}}^{t} \dot{e}_{x}^{T}(s) Z_{1} \dot{e}_{x}(s) \mathrm{d} s+h_{12} \dot{e}_{x}^{T}(t) Z_{2} \dot{e}_{x}(t)-\int_{t-h_{2}}^{t-h_{1}} \dot{e}_{x}^{T}(s) Z_{2} \dot{e}_{x}(s) \mathrm{d} s .
\end{aligned}
$$


From the Leibniz-Newton formula, the following equations are true for any matrices $M^{\prime}$ and $N^{\prime}$ with appropriate dimensions:

$$
2 \eta^{T}(t) M^{\prime}\left[e_{x}(t)-e_{x}(t-\mathrm{d}(l))-\int_{t-d(l)}^{t} \dot{e}_{x}(s) \mathrm{d} s\right]=0,
$$

$$
2 \eta^{T}(t) N^{\prime}\left[e_{x}(t-\mathrm{d}(l))-e_{x}\left(t-h_{2}\right)-\int_{t-h_{2}}^{t-d(l)} \dot{e}_{x}(s) \mathrm{d} s\right]=0,
$$

where $\eta^{T}(t)=\left[e_{x}^{T}(t) e_{x}^{T}(t-d(l)) e_{x}^{T}\left(t-h_{2}\right)\right]$. By adding $(23)$ and (24) to (22), it can obtain that

$$
\begin{aligned}
\mathfrak{J} V\left[e_{x}(t), r, l, t\right] \leq & \eta^{T}(t)\left[\bar{\Pi}(r, l)+h_{2} M^{\prime} Z_{1}^{-1} M^{\prime T}+h_{12} N^{\prime} Z_{2}^{-1} N^{\prime T}+h_{2} \Gamma_{2}^{T} Z_{1} \Gamma_{2}+h_{12} \Gamma_{2}^{T} Z_{2} \Gamma_{2}\right] \eta(t) \\
& +\int_{t-d(l)}^{t}\left[\eta^{T}(t) M^{\prime}+\dot{e}_{x}^{T}(s) Z_{1}\right] Z_{1}^{-1}\left[M^{\prime T} \eta(t)+Z_{1}^{T} \dot{e}_{x}(s)\right] \mathrm{d} s \\
& +\int_{t-h_{2}}^{t-d(l)}\left[\eta^{T}(t) N^{\prime}+\dot{e}_{x}^{T}(s) Z_{2}\right] Z_{2}^{-1}\left[N^{\prime T} \zeta(t)+Z_{2}^{T} \dot{e}_{x}(s)\right] \mathrm{d} s
\end{aligned}
$$

where

$$
\begin{aligned}
\bar{\Pi}(r, l) & =\left[\begin{array}{ccc}
\Xi_{11} & \Xi_{12} & \Xi_{13} \\
* & \Xi_{22} & \Xi_{23} \\
* & * & \Xi_{33}
\end{array}\right], \\
\Gamma_{2}^{T}(r) & =\left[\begin{array}{c}
\widehat{A}^{T}(r) \\
\widehat{A}_{d}^{T}(r) \\
0
\end{array}\right], \\
{\left[M^{\prime} N^{\prime}\right] } & =\left[\begin{array}{ll}
M_{1} & N_{1} \\
M_{2} & N_{2} \\
M_{3} & N_{3}
\end{array}\right] .
\end{aligned}
$$

Because $Z_{i}=Z_{i}^{T}>0(i=1,2)$, then

$$
\left[\eta^{T}(t) M^{\prime}+\dot{e}_{x}^{T}(s) Z_{1}\right] Z_{1}^{-1}\left[M^{\prime T} \eta(t)+Z_{1}^{T} \dot{e}_{x}(s)\right] \geq 0
$$

and $\left[\eta^{T}(t) N^{\prime}+\dot{e}_{x}^{T}(s) Z_{2}\right] Z_{2}^{-1}\left[N^{\prime T} \eta(t)+Z_{2}^{T} \dot{e}_{x}(s)\right] \geq 0$ hold. Therefore, if there exists a feasible solution to $[\bar{\Pi}(r, l)+$ $\left.h_{2} M^{\prime} Z_{1}^{-1} M \quad \prime T+h_{12} N^{\prime} Z_{2}^{-1} N^{\prime T}+h_{2} \Gamma_{2}^{T} Z_{1} \Gamma_{2}+h_{12} \Gamma_{2}^{T} Z_{2} \Gamma_{2}\right]$ $<0$, we can conclude that $\mathfrak{\Im} V\left[e_{x}(t), r, l, t\right]<0$ when $\omega(t)=$ 0 and $f(t)=0$. It is obviously that $\Im V\left[e_{x}(t), r, l, t\right]<0$ can be guaranteed by matrix inequality (14). And, if condition (14) holds, there will exist matrix $\Xi(r, l)>0$, such that $\mathfrak{J} V\left[e_{x}(t), r, l, t\right]=-\eta^{T}(t) \Xi(r, l) \eta(t)<0$. In addition, since $\mathfrak{J} V\left[e_{x}(t), r_{t}, l_{t}, t\right]<0$, we can get $V\left[e_{x}(t), r_{t}, l_{t}, t\right]<V$ $\left.\left[e_{x}(0), r_{0}, l_{0}, t\right]\right|_{t=0}$. Then, by defining $M_{1}=\inf _{-\tau_{2}<\delta_{1}<t}$ $\mathbf{E}\left\{\left\|\eta\left(\delta_{1}\right)\right\|^{2}\right\}, \quad \sigma_{\Xi}=\min _{r \in \Lambda_{1}, l \in \Lambda_{2}} \sigma_{\min }(\Xi(r, l)), \quad$ and $M_{2}=$ $\sup _{-\tau_{2}<\delta_{2}<t} \mathbf{E}\left\{\left\|e_{x}\left(\delta_{2}\right)\right\|^{2}\right\}$, the following relation holds:

$$
\frac{\mathfrak{J} V\left[e_{x}(t), r_{t}, l_{t}, t\right]}{V\left[e_{x}(t), r_{t}, l_{t}, t\right]}<\frac{-\eta^{T}(t) \Xi(r, l) \eta(t)}{\left.V\left[e_{x}(0), r_{0}, l_{0}, t\right]\right|_{t=0}} \leq \frac{-M_{1} \sigma_{\Xi}}{M_{2} \sigma_{\Sigma}}=-\sigma,
$$

where $\sigma_{\Sigma}$ is the sum of a set of positive numbers, $\sigma>0$. So, it follows that $\mathfrak{\Im} V\left[e_{x}(t), r_{t}, l_{t}, t\right]<-\varrho V\left[e_{x}(t), r_{t}, l_{t}, t\right]$. That is, $\mathbf{E}\left\{V\left[e_{x}(t), r_{t}, l_{t}, t\right]\right\}<\exp (-\sigma t) \mathbf{E}\left\{V\left[e_{x}(0), r_{0}, l_{0}, t=0\right]\right\}$.

Then, by letting $\rho=\sigma_{\Sigma} M_{2}$, for a given small positive scalar $\varepsilon>0$, we can get $\varepsilon \mathbf{E}\left\{e_{x}^{T}(t) e_{x}(t)\right\} \leq \mathbf{E}\left\{V\left[e_{x}(t), r_{t}, l_{t}, t\right]\right\} \leq \rho \exp (-\sigma t) . \quad$ Taking limit as $T \longrightarrow \infty$, it follows that

$$
\lim _{T \longrightarrow \infty} \mathbf{E}\left\{\int_{0}^{T} e_{x}^{T}(t) e_{x}(t) \mathrm{d} t \mid e_{x}(0)=\alpha(0), r_{0}, l_{0}\right\} \leq \lim _{t \longrightarrow \infty}\left\{\frac{\rho}{\varepsilon}[1-\exp (-\sigma t)]\right\}=\frac{\rho}{\varepsilon}<\infty .
$$


By recalling Definition 1, we know that the overall global T-S fuzzy error dynamic system (10) is stochastically stable.

Part 3. On the contrary, when $\omega(t) \in L_{2}[0, \infty)$ and $f(t) \neq 0$, to attain the robustness of the proposed observer to the bound disturbances, we define

$$
V_{0}=z^{T}(t) z(t)-\gamma^{2} \omega^{T}(t) \omega(t)+\dot{V}\left[e_{x}(t), r, l, t\right] .
$$

From the Leibniz-Newton formula, the following equations are true for any matrices with appropriate dimensions:

$$
2 \zeta^{T}(t) M\left[e_{x}(t)-e_{x}(t-d(l))-\int_{t-d(l)}^{t} \dot{e}_{x}(s) \mathrm{d} s\right]=0
$$

$$
2 \zeta^{T}(t) N\left[e_{x}(t-d(l))-e_{x}\left(t-h_{2}\right)-\int_{t-h_{2}}^{t-d(l)} \dot{e}_{x}(s) \mathrm{d} s\right]=0,
$$

where $\quad \zeta^{T}(t)=\left[e_{x}^{T}(t) e_{x}^{T}(t-d(l)) e_{x}^{T}\left(t-h_{2}\right) e_{f}^{T}(t) \omega^{T}(t)\right]$, $M=\left[\begin{array}{lllll}M_{1}^{T} & M_{2}^{T} & M_{3}^{T} & 0 & M_{4}^{T}\end{array}\right]^{T}$, and $N=\left[\begin{array}{lllll}N_{1}^{T} & N_{2}^{T} & N_{3}^{T} & 0 & N_{4}^{T}\end{array}\right]^{T}$. By adding (31) and (32) to (30), we can obtain that

$$
\begin{aligned}
V_{0} \leq & e_{x}^{T}(t) L^{\prime T} L^{\prime} e_{x}(t)-\gamma^{2} \omega^{T}(t) \omega(t)+e_{x}^{T}(t)\left[P\left(r_{t}, l_{t}\right) \widehat{A}(r)+\widehat{A}^{T}(r) P\left(r_{t}, l_{t}\right)+\sum_{k=1}^{N_{1}} \pi_{r k} P\left(k_{t}, l_{t}\right)+\sum_{m=1}^{N_{2}} \pi_{l m} P\left(r_{t}, m_{t}\right)\right] \\
e_{x}(t) & +e_{x}^{T}(t)\left(Q+\left(1+\pi h_{12}\right) W\right) e_{x}(t)+2 e_{x}^{T}(t) P(r, l) \widehat{A}_{d}(r) e_{x}\left(t-d(l)+2 e_{x}^{T}(t) P(r, l) \widehat{B}_{f}(r) e_{f}(t)\right)+2 e_{x}^{T}(t) P(r, l) \widehat{D}(r) \omega(t) \\
& -e_{x}^{T}\left(t-h_{2}\right) Q e_{x}\left(t-h_{2}\right)-(1-\dot{d}(l)) e_{x}^{T}(t-d(l)) W e_{x}(t-d(l))-2 e_{f}^{T}(t) \widehat{B}_{f}^{T}(r) P(r, l) \widehat{A}(r) e_{x}(t) \\
& -2 e_{f}^{T}(t) \widehat{B}_{f}^{T}(r) P(r, l) \widehat{A}_{d}(r) e_{x}(t-d(l)) \\
& -2 e_{f}^{T}(t) \widehat{B}_{f}^{T}(r) P(r, l) \widehat{B}_{f}(r) e_{f}(t)-2 e_{f}^{T}(t) \widehat{B}_{f}^{T}(r) P(r, l) \widehat{D}(r) \omega(t)-2 e_{f}^{T}(t) \widehat{B}_{f}^{T}(r) P(r, l) e_{x}(t)+e_{f}^{T}(t) G e_{f}(t)+\delta \\
& +2 \zeta^{T}(t) M\left[e_{x}(t)-e_{x}(t-d(l))-\int_{t-d(l)}^{t} \dot{e}_{x}(s) \mathrm{d} s\right]+2 \zeta^{T}(t) N\left[e_{x}(t-d(l))-e_{x}\left(t-h_{2}\right)-\int_{t-h_{2}}^{t-d(l)} \dot{e}_{x}(s) \mathrm{d} s\right] \\
& +\dot{e}_{x}^{T}(t)\left(h_{2} Z_{1}+h_{12} Z_{2}\right) \dot{e}_{x}(t)-\int_{t-d(l)}^{t} \dot{e}_{x}^{T}(s) Z_{1} \dot{e}_{x}(s) \mathrm{d} s-\int_{t-h_{2}}^{t-d(l)} \dot{e}_{x}^{T}(s) Z_{2} \dot{e}_{x}(s) \mathrm{d} s \\
\leq & \zeta^{T}(t)\left[\Pi(r, l)+h_{2} M Z_{1}^{-1} M^{T}+h_{12} N Z_{2}^{-1} N^{T}+h_{2} \Gamma_{1}^{T} Z_{1} \Gamma_{1}+h_{12} \Gamma_{1}^{T} Z_{2} \Gamma_{1}\right] \zeta(t)+\delta \\
& \left.\left.+\int_{t-d(l)}^{t}\left[\zeta^{T}(t) M+\dot{e}_{x}^{T}(s) Z_{1}\right] Z_{1}^{-1}\left[M^{T} \zeta(t)+Z_{1}^{T} \dot{e}_{x}(s)\right] \mathrm{d} s+\int_{t-h_{2}}^{t-d(l)}\left[\zeta^{T}(t)\right) N+\dot{e}_{x}^{T}(s) Z_{2}\right] Z_{2}^{-1}\left[N^{T} \zeta(t)+Z_{2}^{T} \dot{e}_{x}(s)\right)\right) \mathrm{d} s .
\end{aligned}
$$

Hence, when $\omega(t) \neq 0$ and $f(t) \neq 0$, if there exists a feasible solution to $\zeta^{T}(t)\left[\Pi(r, l)+h_{2} M Z_{1}\right.$ $-1 M^{T}+h_{12} N_{2}^{-1} N^{T}+h_{2} \mathscr{A}_{1}^{T} Z_{1} \mathscr{A}_{1}$ $\left.+h_{12} \mathscr{A}_{1}^{T} Z_{2} \mathscr{A}_{1}\right] \zeta(t)+\delta<0$, it can conclude that

$$
V_{0}=z^{T}(t) z(t)-\gamma^{2} \omega^{T}(t) \omega(t)+\dot{V}<0 .
$$

We assume that $\varepsilon$ is the minimum eigenvalue of $-\left(\Pi(r, l)+h_{2} M Z_{1}^{-1} M^{T} \quad+h_{12} N_{2}^{-1} N^{T}+h_{2} \mathscr{A}_{1}^{T} Z_{1} \mathscr{A}_{1}\right.$ $\left.+h_{12} \mathscr{A}_{1}^{T} Z_{2} \mathscr{A}_{1}\right)$. Therefore, when $\varepsilon\|\zeta(t)\|^{2}>\delta$, equation (34) holds. $\quad \Pi(r, l)+h_{2} M Z_{1}^{-1} M^{T}+h_{12} N_{2}^{-1} N^{T}+h_{2} \mathscr{A}_{1}^{T} Z_{1} \mathscr{A}_{1}$ $+h_{12} \mathscr{A}_{1}^{T} Z_{2} \mathscr{A}_{1}<0$ is equivalent to (14) by Schur complements. This implies the $H_{\infty}$ performance index $J<0$ is satisfied under zero initial condition. The proof is completed.

Remark 3. In order to further reduce the conservativeness of FE criteria for mode-dependent time-varying delay MJSs, two different Markov stochastic processes are considered: one is the state mode $r_{t}$ and the other is time delay mode $l_{t}$. Moreover, when giving FE analysis results for MJSs, the term $\int_{t-h_{2}}^{t} e_{x}^{T}(s) Q e_{x}(s) \mathrm{d} s$ was ignored in [17], or some useful integral term $\int_{-h_{2}}^{-h_{1}} \int_{t+\theta}^{t} \dot{e}_{x}^{T}(s) Z_{2} \dot{e}_{x}(s) \mathrm{d} s \mathrm{~d} \theta$ was lost, e.g., [18].
In this paper, by constructing the new Lyapunov-Krasovskii functional candidate, which make full use of the information of both lower and upper bounds of delay $\mathrm{d}\left(t, l_{t}\right)$, the less conservative result than the existing ones in $[17,18]$ is obtained.

Remark 4. The fast adaptive fault estimation algorithm to estimate the fault has been developed in $[17,20,21]$, but it is not extended to the fuzzy MJSs system with mode-dependent interval time-varying delay, unknown actuator fault, and exogenous disturbance signal simultaneously. If there is only one Markovian process $r_{t}$ and the type of time delay is constant in system, then system (7) in this paper reduces to the existing one in [17]. And, if the fuzzy rule number $s=1,(7)$ can be transformed to the one in [18].

Remark 5. The effect of fuzzy MJSs uncertainties on the estimation errors is decided by the value of $\gamma$. The accuracy of the fault estimation increases with smaller value of $\gamma$. The minimization of $\gamma$ can be found by solving the LMI optimization problem, which has been applied broadly. However, since the term $h_{2} P Z_{1}^{-1} P$ and $h_{12} P Z_{2}^{-1} P$ exist 
simultaneously, Theorem 1 cannot be solved directly. Next, we will choose an inequality transformation method to give the strict LMIs' conditions.

Theorem 2. For the given scalars $\gamma>0$ and $0<h_{1}<h_{2}$, the error dynamic system (10) with mode-dependent time-varying delays is stochastically stable ( when $\omega(t)=f(t)=0$ ) and has a prescribed $H_{\infty}$ performance level $\gamma\left(\right.$ when $\omega(t) \in L_{2}[0, \infty)$ ) if there exist a set of mode-dependent symmetric positivedefinite matrix $P(r, l)>0, Q>0, W>0, Z_{1}>0, Z_{2}>0, G>0$, $H_{i}(r, l), \quad$ and $N=\left[\begin{array}{lllll}N_{1}^{T} & N_{2}^{T} & N_{3}^{T} & 0 & N_{4}^{T}\end{array}\right]$ and $M=\left[\begin{array}{lllll}M_{1}^{T} & M_{2}^{T} & M_{3}^{T} & 0 & M_{4}^{T}\end{array}\right]$, such that the following matrix inequalities hold for all $r, k \in \Lambda_{1}, l, m \in \Lambda_{2}$. Minimize $\rho>0$ which subjects to

$$
\begin{gathered}
{\left[\begin{array}{cc}
\rho I & B_{f q}^{T}(r) P(r, l)-H_{i}(r, l) C_{i}(r) \\
* & \rho I
\end{array}\right]>0,} \\
\Phi_{i i i}(r, l)<0, \quad 1 \leq i \leq s, \\
\Phi_{i j q}(r, l)+\Phi_{j q i}(r, l)+\Phi_{q i j}(r, l)<0, \quad 1 \leq i \leq j<q \leq s, \\
\Phi_{q j i}(r, l)+\Phi_{j i q}(r, l)+\Phi_{i q j}(r, l)<0, \quad 1 \leq i<j \leq q \leq s,
\end{gathered}
$$

where

$$
\begin{aligned}
& \Phi_{i j q}(r, l)=\left[\begin{array}{ccccccccc}
\phi_{11} & \phi_{12} & \Xi_{13} & \phi_{14} & \phi_{15} & h_{2} M_{1} & h_{12} N_{1} & \phi_{18} & \phi_{19} \\
* & \Xi_{22} & \Xi_{23} & \phi_{24} & \Xi_{25} & h_{2} M_{2} & h_{12} N_{2} & \phi_{28} & \phi_{29} \\
* & * & \Xi_{33} & 0 & -N_{4}^{T} & h_{2} M_{3} & h_{12} N_{3} & 0 & 0 \\
* & * & * & \phi_{44} & \phi_{45} & 0 & 0 & \phi_{48} & \phi_{49} \\
* & * & * & * & -\gamma^{2} I & h_{2} M_{4} & h_{12} N_{4} & \phi_{58} & \phi_{59} \\
* & * & * & * & * & -h_{2} Z_{1} & 0 & 0 & 0 \\
* & * & * & * & * & * & -h_{12} Z_{2} & 0 & 0 \\
* & * & * & * & * & * & * & \phi_{88} & 0 \\
* & * & * & * & * & * & * & * & \phi_{99}
\end{array}\right], \\
& \phi_{11}=P(r, l) A_{i}(r)+A_{i}^{T}(r) P(r, l)-Y_{i}(r, l) C_{j}(r)-C_{j}^{T}(r) Y_{i}^{T}(r, l)+\sum_{k=1}^{N_{1}} \Pi_{r k} P(k, l) \\
& +\sum_{m=1}^{N_{2}} \Pi_{l m} P(r, m)+Q+\left(1+\pi h_{12}\right) W+L^{\prime T} L^{\prime}+M_{1}+M_{1}^{T}, \\
& \phi_{12}=P(r, l) A_{d i}(r)-Y_{d i}(r, l) C_{j}(r)-M_{1}+M_{2}^{T}+N_{1}, \\
& \phi_{14}=C_{j}^{T}(r) Y_{i}^{T}(r, l) B_{f q}(r)-A_{i}^{T}(r) P(r, l) B_{f q}(r), \\
& \phi_{15}=P(r, l) D_{i}(r)+M_{4}^{T} \text {, } \\
& \phi_{24}=C_{j}^{T}(r) Y_{d i}^{T}(r, l) B_{f q}(r)-A_{d i}^{T}(r) P(r, l) B_{f q}(r), \\
& \phi_{44}=G-2 B_{f q}^{T}(r) P(r, l) B_{f q}(r) \text {, } \\
& \phi_{45}=-B_{f q}^{T}(r) P(r, l) D_{i}(r), \\
& \phi_{18}=h_{2}\left[A_{i}^{T}(r) P(r, l)-C_{j}^{T}(r) Y_{i}^{T}(r, l)\right], \\
& \phi_{19}=h_{12}\left[A_{i}^{T}(r) P(r, l)-C_{j}^{T}(r) Y_{i}^{T}(r, l)\right], \\
& \phi_{28}=h_{2}\left[A_{d i}^{T}(r) P(r, l)-C_{j}^{T}(r) Y_{d i}^{T}(r, l)\right], \\
& \phi_{29}=h_{12}\left[A_{d i}^{T}(r) P(r, l)-C_{j}^{T}(r) Y_{d i}^{T}(r, l)\right], \\
& \phi_{48}=h_{2} B_{f q}^{T}(r) P(r, l), \\
& \phi_{49}=h_{12} B_{f q}^{T}(r) P(r, l), \\
& \phi_{58}=D_{i}(r) P(r, l) \text {, } \\
& \phi_{59}=D_{i}(r) P(r, l) \text {, } \\
& \phi_{88}=h_{2}\left(-2 \sigma P(r, l)+\sigma^{2} Z_{1}\right) \text {, } \\
& \phi_{99}=h_{12}\left(-2 \sigma P(r, l)+\sigma^{2} Z_{2}\right) \text {. }
\end{aligned}
$$


Moreover, the fast adaptive fault estimation algorithm (11) can realize $e_{x}(t)$ and $e_{f}(t)$ uniformly ultimate bounded and the jumping fault estimation observer gain can be obtained by $L_{i}(r, l)=P-1(r, l) Y_{i}(r, l)$ and $L_{d i}(r, l)=P$ $-1(r, l) Y_{d i}(r, l)$.

Proof. For any scalar $\sigma$, it follows from the fact $\left(\sigma Z_{i}-\right.$ $P(r, l)) Z_{i}^{-1}\left(\sigma Z_{i}-P(r, l)\right) \geq 0$ that

$$
-P(r, l) Z_{i}^{-1} P(r, l) \leq-2 \sigma P(r, l)+\sigma^{2} Z_{i}, \quad(i=1,2) .
$$

Then, considering the error dynamic system (10), we can conclude that condition (14) holds if the following inequality holds:

$$
\sum_{i=1}^{s} \mu_{i}(t) \sum_{j=1}^{s} \mu_{j}(t) \sum_{q=1}^{s} \mu_{q}(t) \Phi_{i j q}(r, l)<0,
$$

where

$$
\begin{aligned}
& \Phi_{i j q}(r, l)=\left[\begin{array}{ccccc}
\Xi(r, l) & h_{2} M^{T} & h_{12} N^{T} & h_{2} \Gamma^{T}(r) P(r, l) & h_{12} \Gamma^{T}(r) P(r, l) \\
* & -h_{2} Z_{1} & 0 & 0 & 0 \\
* & * & -h_{12} Z_{2} & 0 & 0 \\
* & * & * & \phi_{88} & 0 \\
* & * & * & * & \phi_{99}
\end{array}\right] \text {, } \\
& \Xi(r, l)=\left[\begin{array}{ccccc}
\phi_{11} & \phi_{12} & \Xi_{13} & \phi_{14} & \phi_{15} \\
* & \Xi_{22} & \Xi_{23} & \phi_{24} & \Xi_{25} \\
* & * & \Xi_{33} & 0 & -N_{4}^{T} \\
* & * & * & \phi_{44} & \phi_{45} \\
* & * & * & * & -\gamma^{2} I
\end{array}\right] \\
& \widehat{\Gamma}^{T}(r)=\left[\begin{array}{c}
A_{i}^{T}(r) P(r, l)-C_{j}^{T}(r)\left(P(r, l) L_{i}(r, l)\right)^{T} \\
A_{d i}^{T}(r) P(r, l)-C_{j}^{T}(r)\left(P(r, l) L_{d i}(r, l)\right)^{T} \\
0 \\
B_{f q}^{T}(r) P(r, l) \\
D_{i}(r) P(r, l)
\end{array}\right] \\
& \phi_{11}=P(r, l) A_{i}(r)-P(r, l) L_{i}(r, l) C_{j}(r)+A_{i}^{T}(r) P(r, l)-C_{j}^{T}(r) L_{i}^{T}(r, l) P(r, l) \\
& +\sum_{k=1}^{N_{1}} \Pi_{r k} P(k, l)+\sum_{m=1}^{N_{2}} \Pi_{l m} P(r, m)+Q+\left(1+\pi h_{12}\right) W+L^{\prime T} L^{\prime}+M_{1}+M_{1}^{T}, \\
& \phi_{12}=P(r, l) A_{d i}(r)-P(r, l) L_{d i}(r, l) C_{j}(r)-M_{1}+M_{2}^{T}+N_{1} \text {, } \\
& \phi_{14}=C_{j}^{T}(r) L_{i}^{T}(r, l) P(r, l) B_{f q}(r)-A_{i}^{T}(r) P(r, l) B_{f q}(r), \\
& \phi_{15}=P(r, l) D_{i}(r)+M_{4}^{T} \text {, } \\
& \phi_{24}=C_{j}^{T}(r) L_{d i}^{T}(r, l) P(r, l) B_{f q}(r)-A_{d i}^{T}(r) P(r, l) B_{f q}(r), \\
& \phi_{44}=G-2 B_{f q}^{T}(r) P(r, l) B_{f q}(r), \\
& \phi_{45}=-B_{f q}^{T}(r) P(r, l) D_{i}(r) \text {. }
\end{aligned}
$$

It can be easily shown that inequality (41) is equivalent to the following condition:

$$
\begin{aligned}
& \sum_{i=1}^{s} \mu_{i}^{3} \Phi_{i i i}(r, l)+\sum_{i=1}^{s} \mu_{i} \sum_{i \leq j}^{s} \mu_{j} \sum_{j<q}^{s} \mu_{q}\left[\Phi_{i j q}(r, l)+\Phi_{j q i}(r, l)+\Phi_{q i j}(r, l)\right] \\
& +\sum_{i=1}^{s} \mu_{i} \sum_{i<j}^{s} \mu_{j} \sum_{j \leq q}^{s} \mu_{q}\left[\Phi_{q j i}(r, l)+\Phi_{j i q}(r, l)+\Phi_{i q j}(r, l)\right]<0 .
\end{aligned}
$$


By letting $Y_{i}(r, l)=P(r, l) L_{i}(r, l) \quad$ and $Y_{d i}(r, l)=P(r, l) L_{d i}(r, l)$, it leads to the LMIs (36)-(38). On the contrary, it is hard to solve $(36-38)$ with (13) simultaneously, and we can transform it into the optimization problem (35). This completes the proof.

Remark 6. Since condition (13) in Theorem 1 is no longer LMI, it cannot be solved directly using the LMI toolbox of Matlab because of the term $P Z_{i}^{-1} P$. In [20], by defining new variables $S_{i}$ such that $P Z_{i}^{-1} P \geq S_{i}$, an alternative way is proposed to transform the conditions into LMIs based on nonlinear minimization problem using the cone complementary linearization algorithm. However, more new variables $H, U$, and $K_{i}(i=1,2)$ are introduced in the result, which must meet $\left[\begin{array}{ll}U_{i} & H \\ H & K_{i}\end{array}\right] \geq 0, H=P^{-1}, U_{i}=S_{i}^{-1}$, and $K_{i}=Z_{i}^{-1}$. In this paper, by using the inequality transformation method, we can easily obtain the LMIs' conditions without introducing any new variables. This can reduce the computational complexity.

Remark 7. It is necessary to point out that there will bring some difficulties when using Theorem 1 to study the fault estimation observer design problems for fuzzy MJSs due to the existence of equality constraints (13). In order to make $B_{f q}^{T}(r) P(r, l)$ approximate to $H_{i}(r, l) C_{i}(r)$ with a satisfactory precision, a sufficiently small positive scalar $\rho$ should be selected in advance to meet condition (35). We can get the solutions of Theorem 2 by solving an optimization problem with (35), and it is straightforward to check the feasibility of LMIs by using LMIs' toolbox.

Remark 8. You et al. have proposed, in [20], an adaptive observer to estimate the actuator fault-affecting linear systems. The similar work has been extended by He in [17] for T-S fuzzy Markovian jumping systems. The restriction of result in [17] lies in three points. The first one is that the result is about the mode independent state delay. The second one is that the delay must be constant, not considering the interval time delay. The third one is that the external disturbance effect is nonincluded. The main advantage of the proposed design in Theorem 2 is the reduction of conservatism by presenting a mode-dependent time delay result. Also, a time-varying delay has been considered. Furthermore, the design procedure gives the robust fault estimation observer design method.

Remark 9. It should be mentioned that the results proposed in Theorem 2 are built under the conditions that T-S fuzzy systems are about type-1 fuzzy sets and FE observer share the same membership function of the plants. However, in practice, when uncertainties exist in nonlinear plant or the membership functions, the proposed FE method will fail. To overcome this problem, in literature [40], a state feedback nonfragile controller is designed for interval-valued fuzzy systems against nonlinear actuator fault. The obtained results in [40] can better solve the nonfragile control problem for interval-valued fuzzy systems under the condition that different membership functions exist. However, in this situation, how to apply the proposed FE approach to interval-valued T-S fuzzy systems becomes meaningful but also complicated, which will be researched in the future.

\section{Numerical Examples}

In this section, three examples are given to demonstrate the effectiveness of the proposed methods in this paper.

Example 1. We present the following T-S fuzzy MJSs with two jumping operation modes:

$$
\begin{aligned}
& A_{1}(1)=\left[\begin{array}{cc}
-0.5 & -0.3 \\
-0.1 & -1
\end{array}\right], \\
& A_{\tau 1}(1)=\left[\begin{array}{cc}
0.05 & -0.1 \\
0 & -0.05
\end{array}\right], \\
& C_{1}(r)=C_{2}(r)=\left[\begin{array}{ll}
0.6 & 0
\end{array}\right], \\
& A_{1}(2)=\left[\begin{array}{cc}
-0.5 & -0.4 \\
-0.1 & -1.06
\end{array}\right], \\
& A_{\tau 1}(2)=\left[\begin{array}{cc}
0.07 & -0.1 \\
0 & -0.05
\end{array}\right], \\
& D_{1}(r)=D_{2}(r)=\left[\begin{array}{c}
0.5 \\
0
\end{array}\right], \\
& A_{2}(1)=\left[\begin{array}{cc}
-0.5 & -0.3 \\
-0.1 & -1
\end{array}\right], \\
& A_{\tau 2}(1)=\left[\begin{array}{cc}
-0.05 & 0.1 \\
0 & 0.05
\end{array}\right], \\
& B_{1}(r)=B_{2}(r)=\left[\begin{array}{c}
1.0 \\
0.5
\end{array}\right], \\
& A_{2}(2)=\left[\begin{array}{cc}
-0.5 & -0.4 \\
-0.1 & -1.06
\end{array}\right], \\
& A_{\tau 2}(2)=\left[\begin{array}{cc}
-0.07 & 0.1 \\
0 & 0.05
\end{array}\right], \\
& B_{f 2}(r)=\left[\begin{array}{l}
0.1 \\
0.1
\end{array}\right] .
\end{aligned}
$$

In this paper, we assume that $x_{1}(t) \in\left[\begin{array}{ll}-0.5 & 0.5\end{array}\right]$ and represent the membership functions of $h_{1}\left(x_{1}(t)\right)$ and $h_{2}\left(x_{1}(t)\right) \quad$ as $\quad h_{1}\left(x_{1}(t)\right)=0.5\left[1-2 x_{1}(t)\right] \quad$ and $h_{2}\left(x_{1}(t)\right)=0.5\left[1+2 x_{1}(t)\right]$. Meanwhile, we assume state time delay have two changed modes when fuzzy plant rule $i=1=2$, respectively, $d_{1}(t, 1)=d_{2}(t, 1)=0.2 \sin t+0.4$ and $d_{1}(t, 2)=d_{2}(t, 2)=0.5 t+0.3$, so we can get the value $h_{11}=0.2, \quad h_{21}=0.6, \quad h_{12}=0.3$, and $h_{22}=0.8$ and $h_{1}=\min \left\{h_{1 l}, l \in \Lambda_{2}\right\}=0.2$ and $h_{2}=\max \left\{h_{2 l}, l \in \Lambda_{2}\right\}=0.8$ with $\quad \Lambda_{2}=\{1,2\}, \quad \dot{d}_{1}(t, l)=0.2 \cos t \leq h_{31}=0.2$, and $\dot{d}_{2}(t, l)=h_{32}=0.5$. The transition rate matrix that relates the two operation modes are, respectively, given as $\Pi_{1}=\left[\begin{array}{cc}-0.5 & 0.5 \\ 0.4 & -0.4\end{array}\right]$ and $\Pi_{2}=\left[\begin{array}{cc}-0.5 & 0.5 \\ 0.4 & -0.4\end{array}\right]$.

Then, for the fixed scalar $\sigma=1$ and set $\gamma=1.5, \rho=0.001$ applying the solver of LMI toolbox in Matlab, and we can 
obtain the corresponding feasible solutions based on Theorem 2 as follows:

$$
\begin{aligned}
& P(1,1)=\left[\begin{array}{cc}
3.8752 & -0.3151 \\
-0.3151 & 3.8587
\end{array}\right], \\
& P(1,2)=\left[\begin{array}{cc}
3.8320 & -0.3153 \\
-0.3153 & 3.8164
\end{array}\right] \text {, } \\
& P(2,1)=\left[\begin{array}{cc}
3.6467 & -0.3616 \\
-0.3616 & 3.9573
\end{array}\right] \text {, } \\
& P(2,2)=\left[\begin{array}{cc}
3.6508 & -0.3613 \\
-0.3613 & 3.9611
\end{array}\right] \text {, } \\
& Y_{1}(1,1)=\left[\begin{array}{c}
2.2784 \\
-0.6306
\end{array}\right] \text {, } \\
& Y_{d 1}(1,1)=\left[\begin{array}{c}
0.6243 \\
-0.0011
\end{array}\right] \text {, } \\
& Y_{1}(1,2)=\left[\begin{array}{c}
2.2381 \\
-0.6231
\end{array}\right] \text {, } \\
& Y_{d 1}(1,2)=\left[\begin{array}{c}
0.6232 \\
-0.0026
\end{array}\right] \text {, } \\
& Y_{1}(2,1)=\left[\begin{array}{c}
2.0983 \\
-0.6687
\end{array}\right] \text {, } \\
& Y_{d 1}(2,1)=\left[\begin{array}{c}
0.6927 \\
-0.0417
\end{array}\right] \text {, } \\
& Y_{1}(2,2)=\left[\begin{array}{c}
2.1030 \\
-0.6690
\end{array}\right] \text {, } \\
& Y_{d 1}(2,2)=\left[\begin{array}{c}
0.6928 \\
-0.0416
\end{array}\right] \text {, } \\
& Y_{2}(1,1)=\left[\begin{array}{c}
2.1156 \\
-0.6881
\end{array}\right] \text {, } \\
& Y_{d 2}(1,1)=\left[\begin{array}{l}
0.1013 \\
0.1017
\end{array}\right] \text {, } \\
& Y_{2}(1,2)=\left[\begin{array}{c}
2.0816 \\
-0.6770
\end{array}\right] \text {, } \\
& Y_{d 2}(1,2)=\left[\begin{array}{l}
0.1056 \\
0.0998
\end{array}\right] \text {, } \\
& Y_{2}(2,1)=\left[\begin{array}{c}
1.9343 \\
-0.7369
\end{array}\right] \text {, } \\
& Y_{d 2}(2,1)=\left[\begin{array}{c}
-0.0267 \\
0.1021
\end{array}\right] \text {, } \\
& Y_{2}(2,2)=\left[\begin{array}{c}
1.9384 \\
-0.7376
\end{array}\right] \text {, } \\
& Y_{d 2}(2,2)=\left[\begin{array}{c}
-0.0313 \\
0.1022
\end{array}\right] \text {, } \\
& L_{1}(1,1)=\left[\begin{array}{c}
0.5785 \\
-0.1162
\end{array}\right] \text {, }
\end{aligned}
$$

$$
\begin{aligned}
& L_{d 1}(1,1)=\left[\begin{array}{l}
0.1622 \\
0.0130
\end{array}\right], \\
& L_{1}(1,2)=\left[\begin{array}{c}
0.5746 \\
-0.1153
\end{array}\right], \\
& L_{d 1}(1,2)=\left[\begin{array}{l}
0.1637 \\
0.0129
\end{array}\right], \\
& L_{1}(2,1)=\left[\begin{array}{l}
0.5638 \\
-0.1175
\end{array}\right], \\
& L_{d 1}(2,1)=\left[\begin{array}{l}
0.1906 \\
0.0069
\end{array}\right], \\
& L_{1}(2,2)=\left[\begin{array}{l}
0.5644 \\
-0.1174
\end{array}\right], \\
& L_{2}(1,1)=\left[\begin{array}{l}
0.5350 \\
-0.1346
\end{array}\right], \\
& L_{d 1}(2,2)=\left[\begin{array}{l}
0.1905 \\
0.0069
\end{array}\right],
\end{aligned}
$$

$$
L_{d 2}(1,1)=\left[\begin{array}{l}
0.0285 \\
0.0287
\end{array}\right] \text {, }
$$$$
L_{2}(1,2)=\left[\begin{array}{c}
0.5322 \\
-0.1334
\end{array}\right] \text {, }
$$$$
L_{d 2}(1,2)=\left[\begin{array}{l}
0.0299 \\
0.0286
\end{array}\right] \text {, }
$$$$
L_{2}(2,1)=\left[\begin{array}{c}
0.5166 \\
-0.1390
\end{array}\right] \text {, }
$$$$
L_{d 2}(2,1)=\left[\begin{array}{c}
-0.0048 \\
0.0254
\end{array}\right] \text {, }
$$$$
L_{2}(2,2)=\left[\begin{array}{c}
0.5172 \\
-0.1390
\end{array}\right] \text {, }
$$$$
L_{d 2}(2,2)=\left[\begin{array}{c}
-0.0061 \\
0.0252
\end{array}\right] \text {. }
$$

$G=0.0126$, and $H_{i}(r, l)=0.5687(i=1,2)$. Then, by the results of Theorem 2, the observer gains $L_{i}(r, l)$ and $L_{d i}(r, l)$ are obtained as follows:

For the simulation, we assume that the abrupt fault signal is created as 

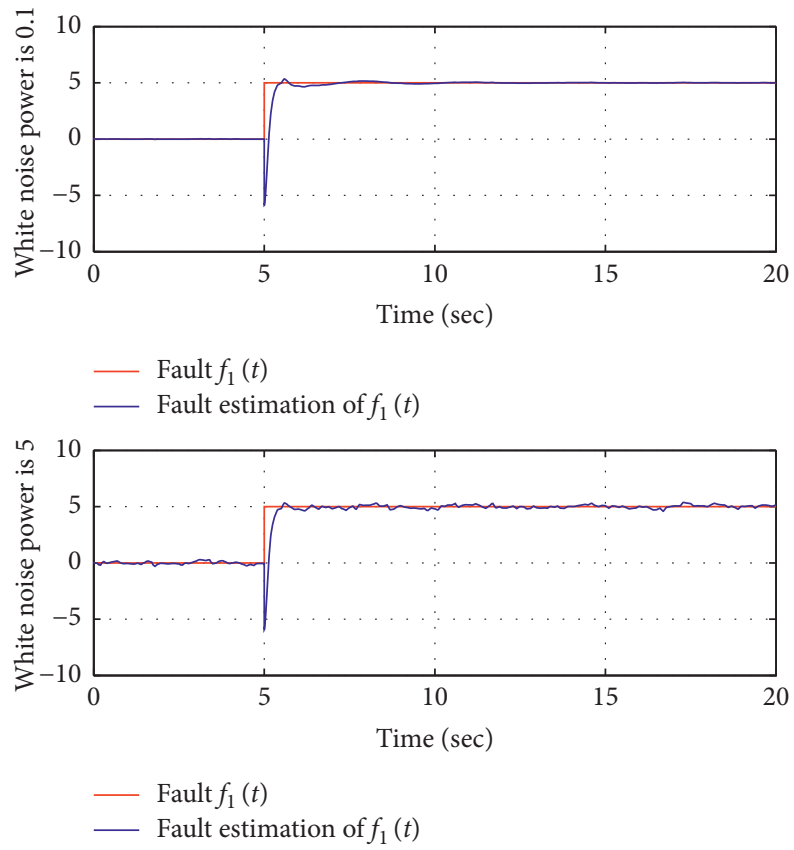

FIgURE 1: Response curves of constant fault $f_{1}(t)$ and its estimation.

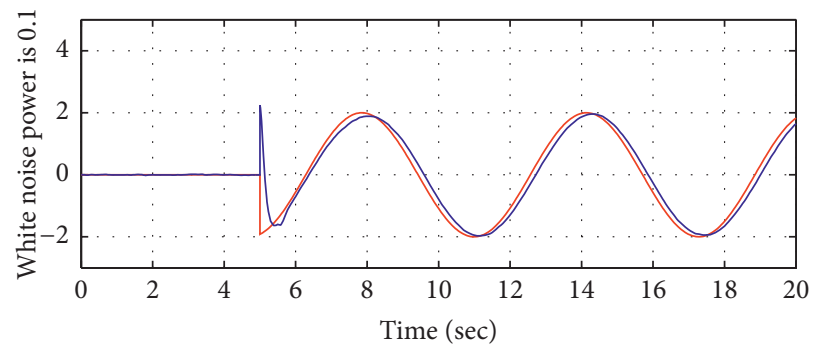

- Fault $f_{2}(t)$

- Fault estimation of $f_{2}(t)$

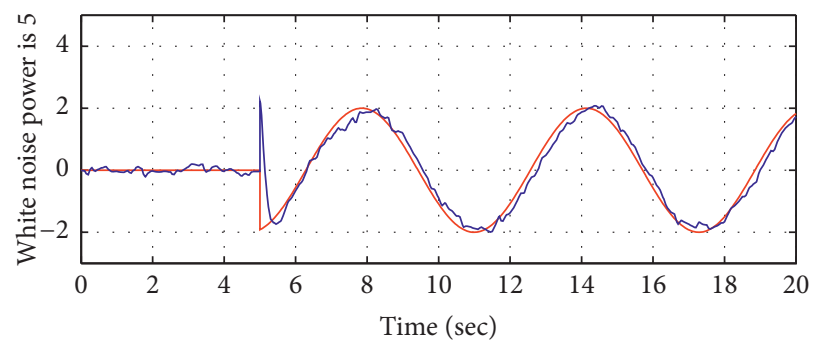

Fault $f_{2}(t)$

Fault estimation of $f_{2}(t)$

Figure 2: Response curves of time-varying fault $f_{2}(t)$ and its estimation.

$$
\begin{aligned}
& f_{1}(t)= \begin{cases}0, & 0 \leq t<5, \\
5, & 5 \leq t \leq 20,\end{cases} \\
& f_{2}(t)= \begin{cases}0 & 0 \leq t<5, \\
2 \sin (t) & 5 \leq t \leq 20 .\end{cases}
\end{aligned}
$$

By taking the learning rate $\Gamma=100$ and assuming $\omega(t)$ is band-limited white noise with sampling time $0.01 \mathrm{~s}$ and noise power is 0.1 and 5, respectively. Such an assumption remains the same for the next simulation examples. Then, using the fault estimation approach in Theorem 2, simulation results shown in Figures 1 and 2 obviously 


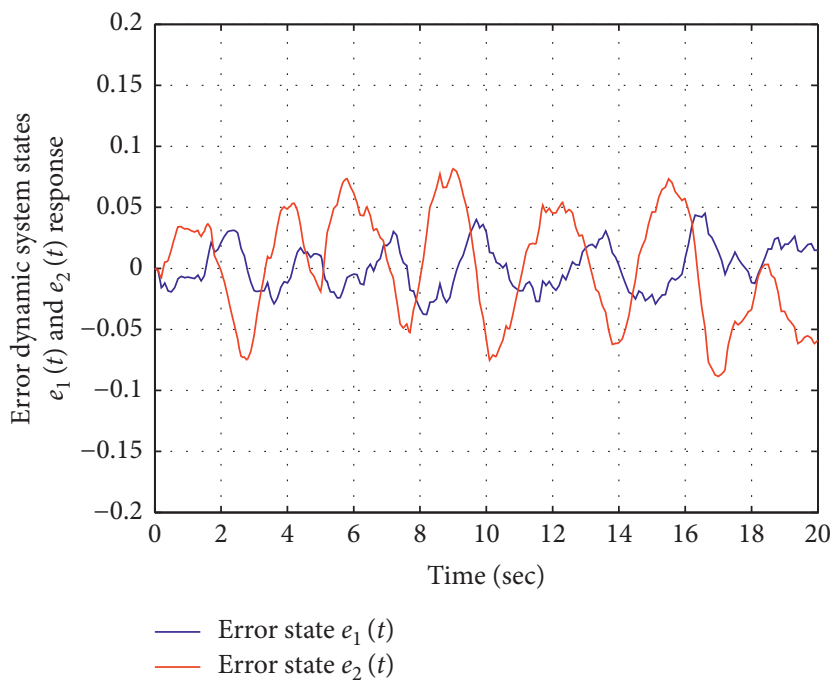

FIgURE 3: Response curves of error dynamic states $e_{1}(t)$ and $e_{2}(t)$ when $\omega(t) \neq 0$.
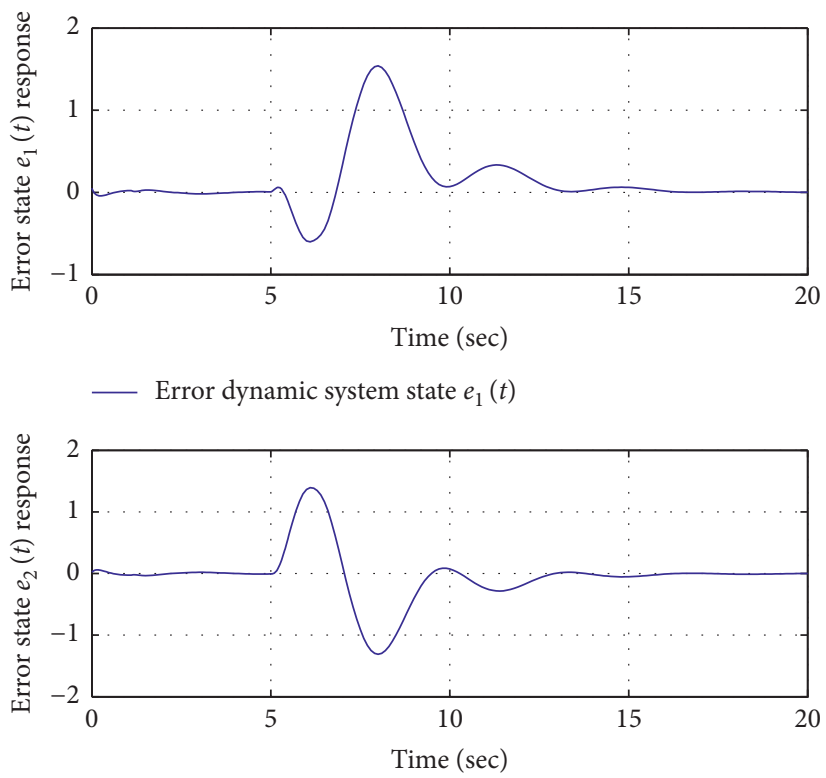

Error dynamic system state $e_{2}(t)$

Figure 4: Response curves of error dynamic states $e_{1}(t)$ and $e_{2}(t)$ when $\omega(t)=0$.

illustrate that the proposed FE method have a good performance to estimate the system fault. Meanwhile, it can be seen from Figures 3 and 4 that the error dynamic system has a small fluctuation when $\omega(t) \neq 0$ and it is also stochastic stable when the disturbance input $\omega(t)$ does not exists. 
Example 2. For comparison purpose, next, we consider a computer simulated truck-trailer system borrowed from $[22,23]$ :

$$
\left\{\begin{array}{l}
\dot{x}_{1}(t)=-\frac{v \bar{t}}{L t_{0}} x_{1}(t)+\frac{v \bar{t}}{l t_{0}} u(t), \\
\dot{x}_{2}(t)=\frac{v \bar{t}}{L t_{0}} x_{1}(t), \\
\dot{x}_{3}(t)=\frac{v \bar{t}}{t_{0}} \sin \left[x_{2}(t)+\frac{v \bar{t}}{2 L} x_{1}(t)\right] .
\end{array}\right.
$$

Then, system $x_{1}(t)$ is perturbed by time delay, and the delayed model is given as

$$
\left\{\begin{array}{l}
\dot{x}_{1}(t)=-a \frac{v \bar{t}}{L t_{0}} x_{1}(t)-(1-a) \frac{v \bar{t}}{L t_{0}} x_{1}(t-\tau(t))+\frac{v \bar{t}}{l t_{0}} u(t), \\
\dot{x}_{2}(t)=a \frac{v \bar{t}}{L t_{0}} x_{1}(t)+(1-a) \frac{v \bar{t}}{L t_{0}} x_{1}(t-\tau(t)) \\
\dot{x}_{3}(t)=\frac{v \bar{t}}{t_{0}} \sin \left[x_{2}(t)+a \frac{v \bar{t}}{2 L} x_{1}(t)+(1-a) \frac{v \bar{t}}{2 L} x_{1}(t-\tau(t))\right] .
\end{array}\right.
$$

The constant $a$ is the retarded coefficient, which satisfies the conditions: $a \in[0,1]$. The limits 1 and 0 correspond to no delay term and to a completed delay term, respectively. In this example, the model parameters is given as $a=0.7$, $l=2.8, L=5.5, v=-1.0, \bar{t}=2.0$, and $t_{0}=0.5$.

Then, we use the following fuzzy models to design the fuzzy fault observer:

Rule 1: IF $\xi_{1}(t)=x_{2}(t)+a(v \bar{t} /(2 L)) x_{1}(t)+(1-a)(v \bar{t} /$ $(2 L)) x_{1}(t-\tau)$ is about 0 , THEN

$$
\left\{\begin{array}{l}
\dot{x}(t)=A_{1} x(t)+A_{d 1} x(t-d(t))+B_{1} u(t), \\
y(t)=C_{1} x(t) .
\end{array}\right.
$$

Rule 2: $\operatorname{IF} \xi_{1}(t)=x_{2}(t)+a(v \bar{t} /(2 L)) x_{1}(t)+(1-a)(v \bar{t}$ $/(2 L)) x_{1}(t-\tau)$ is about $\pi$ or $-\pi$, THEN

$$
\left\{\begin{array}{l}
\dot{x}(t)=A_{2} x(t)+A_{d 2} x(t-d(t))+B_{2} u(t), \\
y(t)=C_{2} x(t) .
\end{array}\right.
$$

Thus, the delay model with fault $f(t)$ and disturbance $\omega(t)$ is given by T-S fuzzy systems as follows:

$$
\left\{\begin{array}{l}
\dot{x}(t)=\sum_{i=1}^{2} \mu_{i}(\xi(t))\left[A_{i} x(t)+A_{d i} x(t-\mathrm{d}(t))+B_{i} u(t)+B_{f i} f(t)+D_{i} \omega(t)\right] \\
y(t)=\sum_{i=1}^{2} \mu_{i}(\xi(t)) C_{i} x(t)
\end{array}\right.
$$

where $x(t)=\left[\begin{array}{lll}x_{1}(t) & x_{2}(t) & x_{3}(t)\end{array}\right]^{T}$ and 


$$
\begin{aligned}
& A_{1}=\left[\begin{array}{ccc}
-a \frac{v \bar{t}}{L t_{0}} & 0 & 0 \\
a \frac{v \bar{t}}{L t_{0}} & 0 & 0 \\
a \frac{v^{2} \bar{t}^{2}}{2 L t_{0}} & \frac{v \bar{t}}{t_{0}} & 0
\end{array}\right], \\
& A_{d 1}=\left[\begin{array}{ccc}
-(1-a) \frac{v \bar{t}}{L t_{0}} & 0 & 0 \\
(1-a) \frac{v \bar{t}}{L t_{0}} & 0 & 0 \\
(1-a) \frac{v^{2} \bar{t}^{2}}{2 L t_{0}} & 0 & 0
\end{array}\right] \text {, } \\
& B_{1}=\left[\begin{array}{c}
\frac{v \bar{t}}{l t_{0}} \\
0 \\
0
\end{array}\right] \text {, } \\
& A_{2}=\left[\begin{array}{ccc}
-a \frac{v \bar{t}}{L t_{0}} & 0 & 0 \\
a \frac{v \bar{t}}{L t_{0}} & 0 & 0 \\
a \frac{d v^{2} \bar{t}^{2}}{2 L t_{0}} & \frac{d v \bar{t}}{t_{0}} & 0
\end{array}\right], \\
& A_{d 2}=\left[\begin{array}{ccc}
-(1-a) \frac{v \bar{t}}{L t_{0}} & 0 & 0 \\
(1-a) \frac{v \bar{t}}{L t_{0}} & 0 & 0 \\
(1-a) \frac{d v^{2} \bar{t}^{2}}{2 L t_{0}} & 0 & 0
\end{array}\right] \text {, } \\
& B_{2}=\left[\begin{array}{c}
\frac{v \bar{t}}{l t_{0}} \\
0 \\
0
\end{array}\right] .
\end{aligned}
$$

Here, because the actuator faults usually occur in the input channel, we assume that $B_{i}=B_{f i}(i=1,2)$ and $C_{1}=$ $C_{2}=\left[\begin{array}{lll}-2 & 0.05 & -0.15\end{array}\right]$ and the disturbance distribution matrices are $D_{1}=D_{2}=\left[\begin{array}{lll}0.05 & 0 & 0\end{array}\right]^{T}$. For simulation purpose, we choose membership functions for Rules 1 and 2 are $\mu_{1}(\xi(t))=\left(1 /\left(1+\exp \left(x_{1}(t)+0.5\right)\right)\right) \quad$ and $\quad \mu_{2}(\xi(t))=1-$ $\mu_{1}(\xi(t))$ with initial condition $\left[\begin{array}{llll}0.5 \pi & 0.75 \pi & -5\end{array}\right]^{T}$ and set $d=10 *\left(t_{0} / \pi\right)$.

It should be noted that the abovementioned time-delay fuzzy system is a special case that we considered in this paper, which do not have mode change both system matrices and state delay under different fuzzy rules. So, we assume the time-varying delay acting on the system state is given by $\mathrm{d}(t)=0.2 \sin (t)+0.3\left(h_{1}=0.1, h_{2}=0.5, h_{12}=0.4, h_{3}=\right.$ $0.2)$ which are the same as [22]; then, by computing matrix inequalities (35)-(38) in Theorem 2 based on Matlab LMIs toolbox, one obtains a feasible solution when $\gamma=3$ :

$$
\begin{aligned}
& Y_{1}=\left[\begin{array}{c}
0.0473 \\
-0.0850 \\
-0.003
\end{array}\right] \text {, } \\
& Y_{d 1}=\left[\begin{array}{c}
0.0058 \\
-0.0369 \\
-0.0001
\end{array}\right] \text {, } \\
& Y_{2}=\left[\begin{array}{c}
0.0522 \\
-0.0933 \\
-0.0003
\end{array}\right] \text {, } \\
& Y_{d 2}=\left[\begin{array}{c}
0.0063 \\
-0.0405 \\
-0.0001
\end{array}\right] \text {, } \\
& L_{1}=\left[\begin{array}{c}
3.2976 \\
-0.9125 \\
-1.8335
\end{array}\right] \text {, } \\
& L_{d 1}=\left[\begin{array}{c}
0.4022 \\
-0.3999 \\
0.3674
\end{array}\right] \text {, } \\
& L_{2}=\left[\begin{array}{c}
3.6335 \\
-1.0041 \\
-1.4626
\end{array}\right] \text {, } \\
& L_{d 2}=\left[\begin{array}{c}
0.4364 \\
-0.4401 \\
0.6833
\end{array}\right] \text {. }
\end{aligned}
$$

Then, a time-varying fault is simulated as

$$
f(t)= \begin{cases}0, & 0 \leq t<5, \\ \sin (3 t-9)+\sin (4 t-12), & 5 \leq t \leq 20 .\end{cases}
$$

Figure 5 illustrates fault estimation simulation results. It is obvious that despite the existing initial error, the fault is estimated with satisfactory accuracy and rapidity with time. It follows from Figure 6 that the error states $e_{x_{1}}(t), e_{x_{2}}(t)$, and $e_{x_{3}}(t)$ are also stable when $\omega(t)$ not exists. On the contrary, the estimation error shown in Figure 7 fluctuates in a limited range when $\omega(t) \neq 0$. 
Remark 10. In the existing literature [22], by means of the adaptive observer, the robust fault estimation for time delay T-S fuzzy systems is solved. However, it is worth mentioning that the works in [22] have considered no mode change case that the system matrices and time delay have fixed value under different fuzzy rules. However, this is not the situation always because, in practice, the fixed value cannot describe the system well, and the descriptions with mode changes are more general. Consider this characteristic of system, and the FE strategy in [22] is not valid. From Figure 5, we can see that, on one hand, the proposed method can accurately realize fault estimation under disturbance input despite there being an error in the initial stage of fault estimation. It means that whether the system and delay have mode change or not, the fault estimator always works to give the fault estimation information.

Remark 11. On the contrary, free-weighting matrix method is a common method when dealing with integral term of the derivative of Lyapunov functional candidate; however, what followed is the increasing of computational complexity. In [22], there are 18 unknown matrices in Theorem 3.1 to be solved, and they are $P, Q_{1}, Q_{2}, G, R, Z, L, \bar{R}, M_{i}$, and $N_{i}$, $i=1,2,3,4,5$, where $M_{i}$ and $N_{i}$ are free-weighting matrix. The higher the dimension of the system, the more the variables of LMIs. If mode switching is not considered, there are 15 unknown matrices in Theorem 2 including freeweighting matrices $M_{i}$ and $N_{i}, i=1,2,3,4$. Thus, by combining with the model and calculation of characteristics of the proposed approach, we can see that the multimode change FE method is more simpler and can be used in more field of application.

Remark 12. Generally speaking, a large number of fuzzy rules are usually needed for accurate approximation, but which may lead inefficiency or difficulty in system computation. When the number of fuzzy rules is increased, the number of LMIs to be solved increases at the same time. If fuzzy rules $r=2$, there are eight LMIs in Theorem 3.1 of literature 22. However, only five LMIs are required to obtain the gain matrices by using the proposed approach in this paper. When $r=3$ and $r=4$, there are 15 and 24 LMIs to be solved in Theorem 3.1. In Theorem 2, we just need to calculate 11 and 19 LMIs, respectively. From the comparison results in Table 1, we can see that fewer decision variables and LMIs to be solved will reduce the computational complexity.

Example 3. We consider the following example of a singlelink robot arm model presented in [11]:

$$
\ddot{\theta}(t)=-\frac{M g l}{J} \sin (\theta(t))-\frac{D}{J} \dot{\theta}(t)+\frac{1}{J} u(t)+\frac{L}{J} \omega(t),
$$

where $\theta(t)$ is the angle position of the arm, $u(t)$ is the control input, $\omega(t)$ is the unknown disturbance, $M$ is the mass of the payload, $g$ is the acceleration of gravity, $L$ is the length of the arm, $J$ is the moment of inertia, and $D$ is the coefficient of viscous friction. The values of parameters $g, D$, and $L$ are given by $g=9.8, D=0.2$, and $L=0.5$, respectively. It is also supposed that the system parameters $M$ and $J$ have two different modes as [17], when system mode $r=1$, set $M=1$ and $J=1$, and when system mode $r=2$, we set $M=5$ and $J=5$. And, the transition rate matrices that relate the two operation modes are given as

$$
\begin{aligned}
& \Pi_{1}=\left[\begin{array}{cc}
-0.5 & 0.5 \\
0.4 & -0.4
\end{array}\right], \\
& \Pi_{2}=\left[\begin{array}{cc}
-0.8 & 0.8 \\
0.6 & -0.6
\end{array}\right] .
\end{aligned}
$$

Here, we let the system state $x(t)=\left(x_{1}(t), x_{2}(t)\right)$ $=(\theta(t), \dot{\theta}(t))$, where $x_{2}(t)$ denotes the time rate change of the angle position of arm. When $\theta(t)$ changes, $x_{2}(t)=\dot{\theta}(t)$ will change accordingly. However, in this process, there will be undesired phenomena, such as time delay. In [41], it is assumed that $x_{2}(t)$ is perturbed by one fixed type timevarying delay to study the $H_{\infty}$ control problem. However, there will be a certain degree inaccuracy by means of this kind of time delays. In order to better describe the randomness of time delay, it is necessary to consider the modedependent time delays when single-link robot arm system modes switch randomly from one mode to another. Under the condition $-179.4270<\theta(t)<179.4270$, the nonlinear term $\sin (\theta(t))$ can be represented as $\sin \left(x_{1}(t)\right)=h_{1}$ $\left(x_{1}(t)\right)+h_{2}\left(x_{1}(t)\right) \lambda x_{1}(t)$, with $\lambda=(1 / 100 \pi)$, where $h_{1}\left(x_{1}(t)\right), \quad h_{2}\left(x_{1}(t)\right) \in[0,1], \quad$ and $\quad h_{1}\left(x_{1}(t)\right)+h_{2}\left(x_{1}\right.$ $(t))=1$. Then, using the membership function $h_{1}\left(x_{1}(t)\right)$ and $h_{2}\left(x_{1}(t)\right)$, we can get the time-delay nonlinear MJSs with actuator fault by the following T-S models for the single-link robot arm with the selected parameter $\lambda$ :

Plant rule 1: IF $x_{1}(t)$ is about $0 \mathrm{rad}$, THEN

$$
\begin{aligned}
\dot{x}(t)= & A_{1}(r) x(t)+A_{d 1}(r) x(t-\mathrm{d}(t, l))+B_{1}(r) u(t) \\
& +B_{f 1}(r) f(t)+D_{1}(r) \omega(t) .
\end{aligned}
$$

Plant rule 2: IF $x_{1}(t)$ is about $\pi \operatorname{rad}$ or $-\pi \mathrm{rad}$, THEN

$$
\dot{x}(t)=A_{2}(r) x(t)+A_{d 2}(r) x(t-d(t, l))+B_{2}(r) u(t)+B_{f 2}(r) f(t)+D_{2}(r) \omega(t),
$$

where the system matrices of two different modes are as follows: 


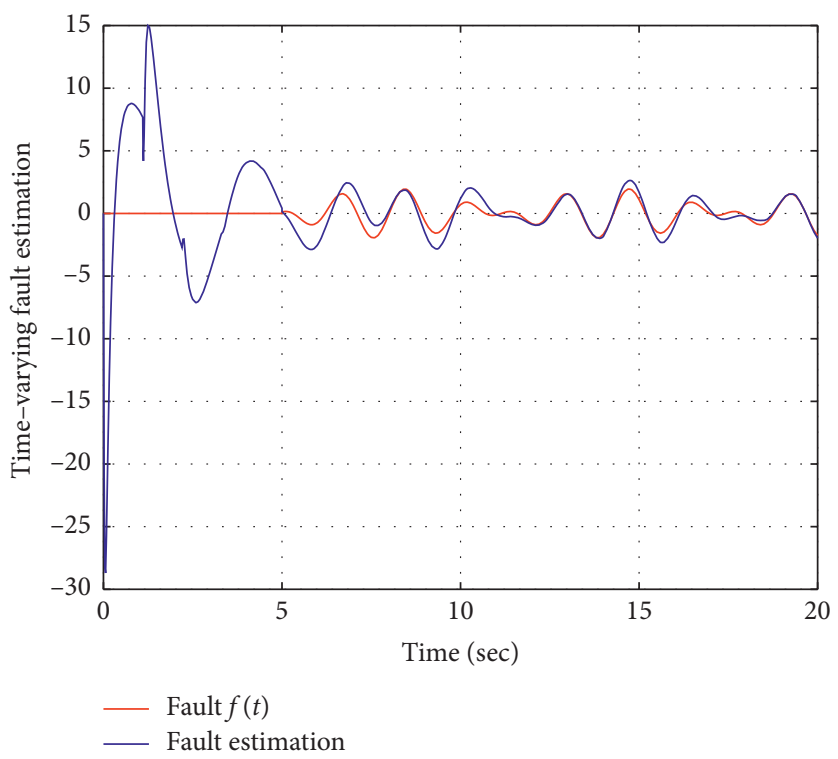

FIgURE 5: Response curves of time-varying fault $f(t)$ and its estimation.
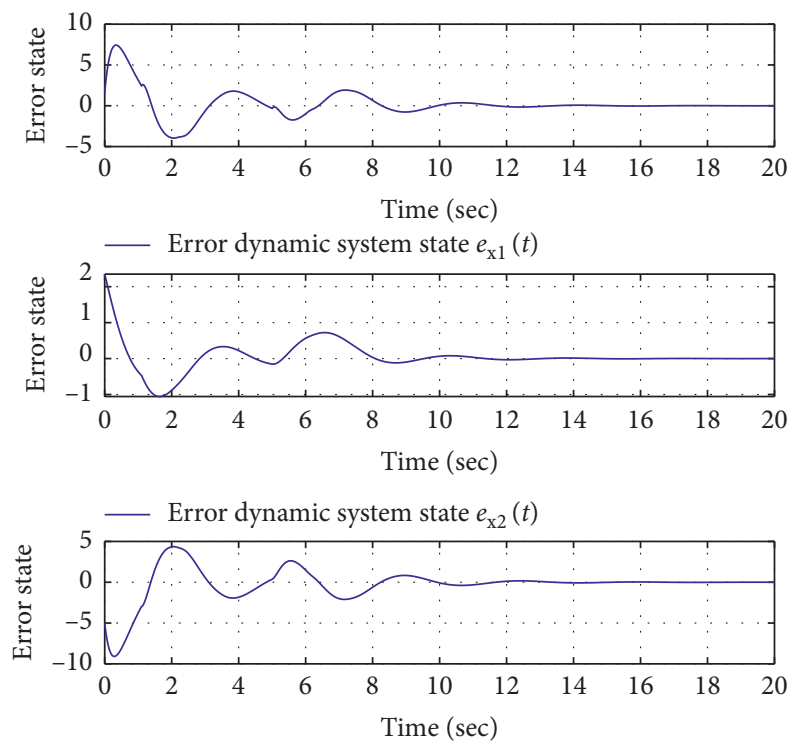

_ Error dynamic system state $e_{\mathrm{x} 3}(t)$

FIGURE 6: Response curves of error dynamic states $e_{x_{1}}(t), e_{x_{2}}(t)$, and $e_{x_{3}}(t)$ when $\omega(t)=0$. 


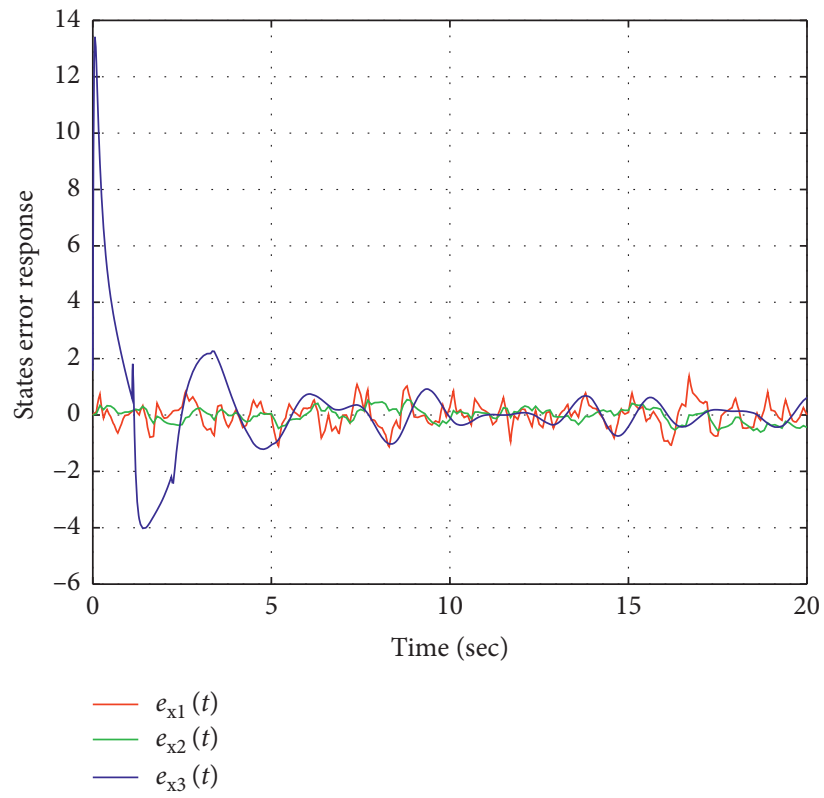

Figure 7: Response curves of error dynamic states $e_{x_{1}}(t), e_{x_{2}}(t)$, and $e_{x_{3}}(t)$ when $\omega(t) \neq 0$.

TABLE 1: Comparison of the number of variables and LMIs to be solved.

The number of variables and LMIs to be solved

Fuzzy rules

[40] $r=2$

$r=3$

$r=4$

Decision variables

Our results

Reduction ratio

$37.5 \%$

15
11

24

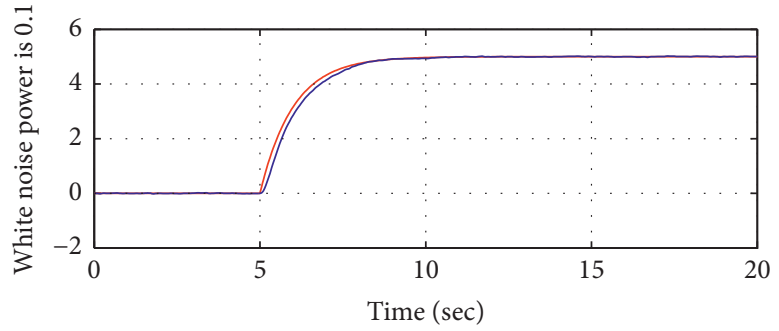

Fault $f_{3}(t)$

- Fault estimation of $f_{3}(t)$

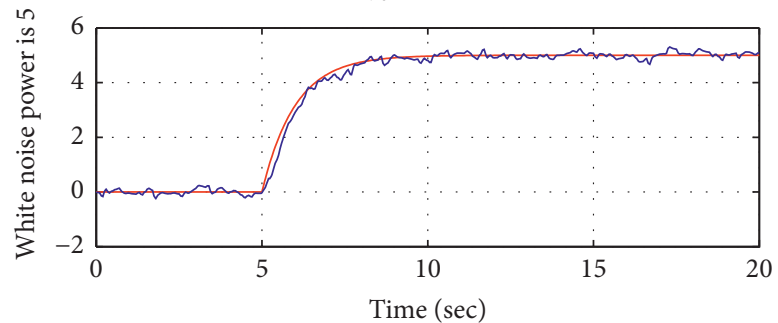

Fault $f_{3}(t)$

Fault estimation of $f_{3}(t)$

FIgURE 8: Response curves of constant fault $f_{3}(t)$ and its estimation. 


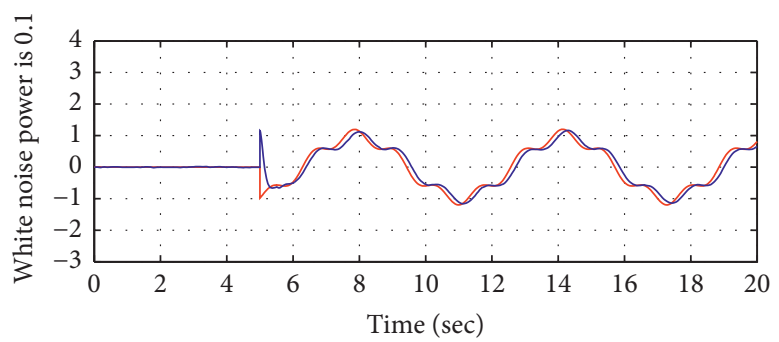

— Fault $f_{4}(t)$

- Fault estimation of $f_{4}(t)$

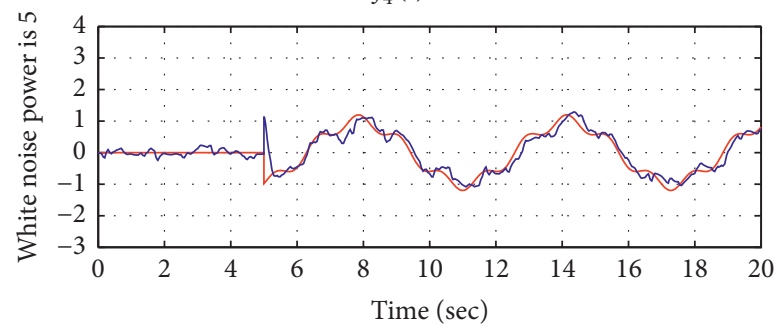

- Fault $f_{4}(t)$

- Fault estimation of $f_{4}(t)$

FIgURE 9: Response curves of time-varying fault $f_{4}(t)$ and its estimation.

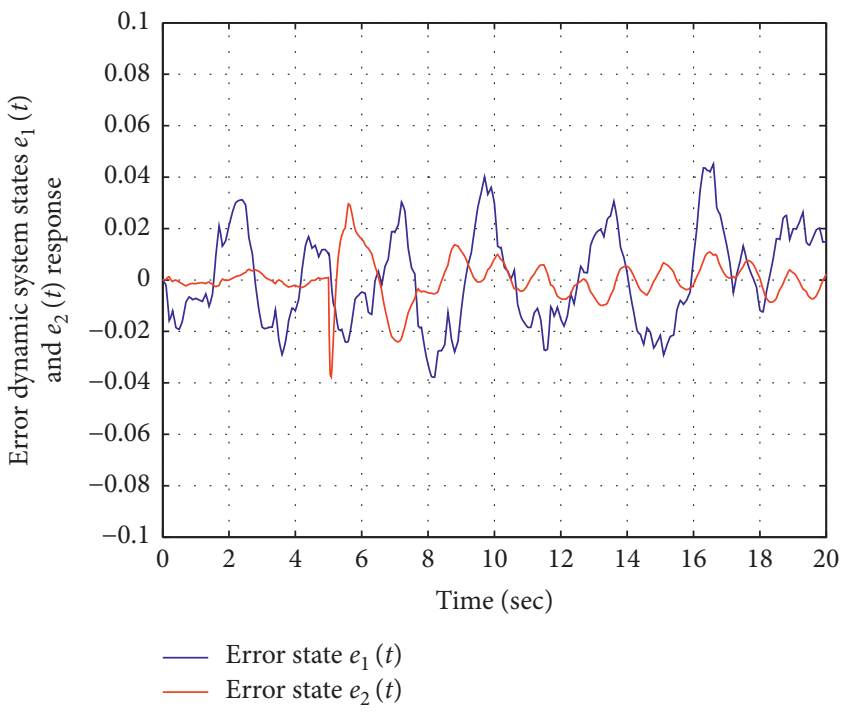

Figure 10: Response curves of error dynamic states $e_{1}(t)$ and $e_{2}(t)$ when $\omega(t) \neq 0$. 


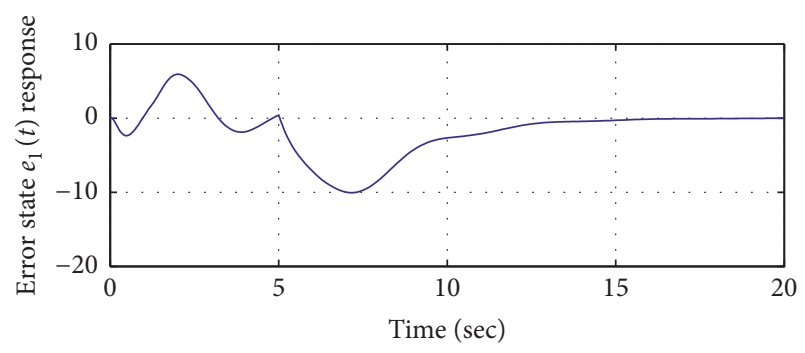

when the attenuation value $\gamma=1.5$, one can obtain the feasible solutions by computing the matrix inequalities (35)-(38) in Theorem 2.

When $i=1$,

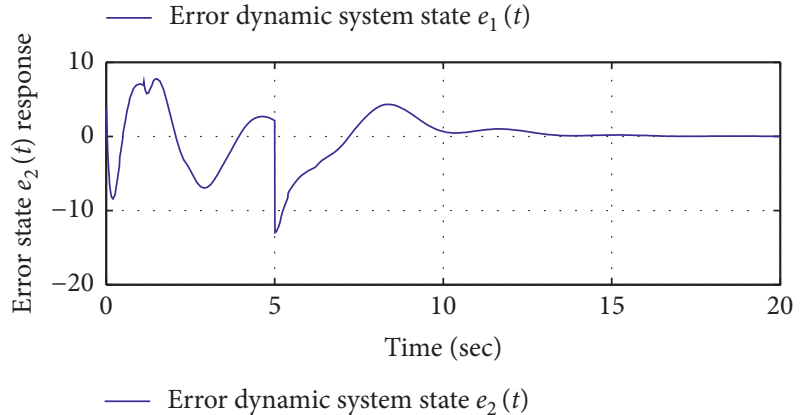

FIGURE 11: Response curves of error dynamic states $e_{1}(t)$ and $e_{2}(t)$ when $\omega(t)=0$.

$$
\left.\begin{array}{rl}
A_{1}(1) & =\left[\begin{array}{cc}
0 & 1 \\
-g L & -D
\end{array}\right], \\
A_{d 1}(1) & =\left[\begin{array}{cc}
0.05 & -0.1 \\
0 & -0.05
\end{array}\right], \\
B_{1}(1) & =B_{2}(1)=\left[\begin{array}{c}
1 \\
0.5
\end{array}\right], \\
A_{1}(2) & =\left[\begin{array}{cc}
0 & 1 \\
-g L & -0.8 D
\end{array}\right], \\
A_{d 1}(2) & =\left[\begin{array}{cc}
0.07 & -0.1 \\
0 & -0.05
\end{array}\right], \\
B_{f 1}(1) & =B_{f 2}(1)=\left[\begin{array}{c}
0 \\
0.5
\end{array}\right], \\
A_{2}(1) & =\left[\begin{array}{cc}
0 & 1 \\
-\lambda g L & -D
\end{array}\right], \\
A_{d 2}(1) & =\left[\begin{array}{cc}
0.05 & -0.1 \\
0 & -0.05
\end{array}\right], \\
A_{d 2}(2) & =\left[\begin{array}{cc}
0.07 & -0.1 \\
0 & -0.05
\end{array}\right], \\
B_{1}(2) & =B_{2}(2)=\left[\begin{array}{c}
1 \\
0.5
\end{array}\right], \\
A_{2}(2) & =\left[\begin{array}{cc}
0 & 1 \\
-g L & -0.8 D
\end{array}\right],
\end{array}\right],
$$

$C_{1}(r)=C_{2}(r)=\left[\begin{array}{ll}0.6 & 0\end{array}\right]$, and $D_{1}(r)=D_{2}(r)=\left[\begin{array}{ll}0.1 & 0\end{array}\right]^{T}$. With the initial value for $G=0.5, \sigma=10$, and $\rho=0.001$ and the bounds of time delay remaining the same as example 1 ,

$$
\begin{aligned}
Y_{1}(1,1) & =\left[\begin{array}{c}
31.7772 \\
-8.6152
\end{array}\right], \\
Y_{d 1}(1,1) & =\left[\begin{array}{c}
0.8943 \\
-0.0723
\end{array}\right], \\
Y_{1}(1,2) & =\left[\begin{array}{c}
31.4923 \\
-8.6142
\end{array}\right], \\
Y_{d 1}(1,2) & =\left[\begin{array}{c}
0.8873 \\
-0.0723
\end{array}\right], \\
Y_{1}(2,1) & =\left[\begin{array}{c}
31.0695 \\
-8.5915
\end{array}\right], \\
Y_{d 1}(2,1) & =\left[\begin{array}{c}
0.9397 \\
-0.0648
\end{array}\right], \\
Y_{1}(2,2) & =\left[\begin{array}{c}
31.0937 \\
-8.5915
\end{array}\right], \\
Y_{d 1}(2,2) & =\left[\begin{array}{c}
0.9413 \\
-0.0649
\end{array}\right] .
\end{aligned}
$$

When $i=2$,

$$
\begin{aligned}
Y_{2}(1,1) & =\left[\begin{array}{l}
268.9393 \\
24.5274
\end{array}\right], \\
Y_{d 2}(1,1) & =\left[\begin{array}{c}
-2.8653 \\
-0.2155
\end{array}\right], \\
Y_{2}(1,2) & =\left[\begin{array}{c}
268.6682 \\
24.4924
\end{array}\right], \\
Y_{d 2}(1,2) & =\left[\begin{array}{c}
-2.8622 \\
-0.2152
\end{array}\right], \\
Y_{2}(2,1) & =\left[\begin{array}{c}
282.2325 \\
25.6010
\end{array}\right], \\
Y_{d 2}(2,1) & =\left[\begin{array}{c}
-4.8811 \\
-0.3422
\end{array}\right], \\
Y_{2}(2,2) & =\left[\begin{array}{c}
269.1980 \\
22.0768
\end{array}\right], \\
Y_{d 2}(2,2) & =\left[\begin{array}{c}
-3.3712 \\
-0.2226
\end{array}\right] .
\end{aligned}
$$

Then, by Theorem 2 , the observer gains $L_{i}(r, l)$ and $L_{d i}(r, l)$ are obtained as follows.

When $i=1$, 


$$
\begin{aligned}
L_{1}(1,1) & =\left[\begin{array}{l}
4.7273 \\
4.4522
\end{array}\right], \\
L_{d 1}(1,1) & =\left[\begin{array}{c}
0.0996 \\
-0.0325
\end{array}\right], \\
L_{1}(1,2) & =\left[\begin{array}{c}
4.7270 \\
4.4491
\end{array}\right], \\
L_{d 1}(1,2) & =\left[\begin{array}{c}
0.0997 \\
-0.0325
\end{array}\right], \\
L_{1}(2,1) & =\left[\begin{array}{c}
4.7130 \\
4.6485
\end{array}\right], \\
L_{d 1}(2,1) & =\left[\begin{array}{c}
0.1384 \\
-0.0308
\end{array}\right], \\
L_{1}(2,2) & =\left[\begin{array}{c}
4.7056 \\
4.3736
\end{array}\right], \\
L_{d 1}(2,2) & =\left[\begin{array}{c}
0.1425 \\
-0.0278
\end{array}\right] .
\end{aligned}
$$

When $i=2$,

$$
\begin{aligned}
L_{2}(1,1) & =\left[\begin{array}{l}
4.7699 \\
4.4474
\end{array}\right], \\
L_{d 2}(1,1) & =\left[\begin{array}{l}
-0.0524 \\
-0.0222
\end{array}\right], \\
L_{2}(1,2) & =\left[\begin{array}{l}
4.7692 \\
4.4447
\end{array}\right], \\
L_{d 2}(1,2) & =\left[\begin{array}{l}
-0.0523 \\
-0.0222
\end{array}\right], \\
L_{2}(2,1) & =\left[\begin{array}{l}
4.7515 \\
4.6431
\end{array}\right], \\
L_{d 2}(2,1) & =\left[\begin{array}{l}
-0.0856 \\
-0.0238
\end{array}\right], \\
L_{2}(2,2) & =\left[\begin{array}{l}
4.7340 \\
4.3726
\end{array}\right], \\
L_{d 2}(2,2) & =\left[\begin{array}{l}
-0.0616 \\
-0.0155
\end{array}\right] .
\end{aligned}
$$

For the simulation, we assume that constant fault $f_{3}(t)$ and time-varying fault $f_{4}(t)$ are, respectively, created as

$$
\begin{aligned}
& f_{3}(t)= \begin{cases}0, & 0 \leq t<5, \\
5\left(1-e^{-(t-5)}\right), & 5 \leq t \leq 20\end{cases} \\
& f_{4}(t)= \begin{cases}0, & 0 \leq t<5, \\
\frac{1}{5} \sin (0.5 t)+\sin (t), & 5 \leq t \leq 10 .\end{cases}
\end{aligned}
$$

It can be seen from Figures 8 and 9 that whether the faults are constant or time-varying, the estimation of system faults can be obtained accurately and timely. When the disturbance input exist $\omega(t) \neq 0$, the error dynamic system states fluctuate in a small range which are shown in the simulation result Figure 10, and when $\omega(t)=0$, the stochastic stability of the error system is guaranteed in Figure 11 . The purpose of simulation is to verify the robustness of the proposed method with respect to modeling fault estimation. In order to give a comparison with the small disturbance fault estimate, relatively larger disturbance power is given in Examples 1 and 3 and the other parameters remain the same as before. As indicated by the simulation results shown in Figures 1 and 2 and Figures 8 and 9, we can see that the proposed method can realize accurate fault estimation under disturbance input. This illustrates the effectiveness of the approach proposed in this paper.

\section{Conclusion}

In this paper, we have studied the design of adaptive fault estimation observer for a class of T-S fuzzy Markovian jump systems with mode-dependent interval time-varying delays. By selecting the appropriate Lyapunov-Krasovskii functional and applying LMIs optimization techniques, sufficient conditions are derived such that the error dynamic are stochastically stable and the designed observer ensures the rapidity and accuracy performance of fault estimation. Simulation results illustrate the effectiveness of the proposed approach. It should be noted that the approach presented in this paper requires that the FE observer have the same membership functions as the plants model and not consider the interval-valued fuzzy systems. Nevertheless, the uncertainties exist in membership functions may lead to the inefficiency of the proposed FE methods. In addition, how to solve the fault-tolerant control problem under such conditions becomes particularly important. We will consider these problems in our future work.

\section{Data Availability}

The relevant data of calculation used to support the findings of this study are included within the article.

\section{Conflicts of Interest}

The authors declare that they have no conflicts of interest.

\section{Acknowledgments}

This work was supported in part by the Funds of National Science of China, under Grant 61773013, and Natural Science Foundation of Liaonig Province, China, under Grant 2019-ZD-0276.

\section{References}

[1] N. S. D. Arrifano and V. A. Oliveira, "Robust \$H_\{linfty\}\$ fuzzy control approach for a class of markovian jump nonlinear systems," IEEE Transactions on Fuzzy Systems, vol. 14, no. 6, pp. 738-754, 2006.

[2] H. Ning and K. Cai, "Mode-independent robust stabilization for uncertain Markovian jump nonlinear systems via fuzzy control," IEEE Transaction Systems Man Cybernetwork, vol. 36, no. 3, pp. 509-519, 2006. 
[3] W. Asswinchaichote, S. K. Nguang, and P. Shi, "Robust $H_{\infty}$ fuzzy filter design for uncertain nonlinear singularly perturbed systems with Markovian jumps: an LMI approach," Information Sciences, vol. 177, no. 7, pp. 1699-1714, 2007.

[4] H. Li, H. Gao, P. Shi, and X. Zhao, "Fault-tolerant control of Markovian jump stochastic systems via the augmented sliding mode observer approach," Automatica, vol. 50, no. 7, pp. 1825-1834, 2014.

[5] L. Wu, X. Su, and P. Shi, "Sliding mode control with bounded gain performance of Markovian jump singular time-delay systems," Automatica, vol. 48, no. 8, pp. 1929-1933, 2012.

[6] H. R. Karimi, "A sliding mode approach to Ho synchronization of master-slave time-delay systems with Markovian jumping parameters and nonlinear uncertainties," Journal of the Franklin Institute, vol. 349, no. 4, pp. 1480-1496, 2012.

[7] T. Takagi and M. Sugeno, "Fuzzy identification of systems and its applications to modeling and control," IEEE Transactions on Systems, Man, and Cybernetics, vol. SMC-15, no. 1, pp. 116-132, 1985.

[8] M. Liu, X. Liu, and Y. Shi, "T-S fuzzy-model-based and filtering for networked control systems with two-channel Markovian random delays," Digital Signal Processing, vol. 27, pp. 167-174, 2014.

[9] M. Sathishkumar, R. Sakthivel, O. M. Kwon, and B. Kaviarasan, "Finite-time mixed $\mathrm{H} \infty$ and passive filtering for Takagi-Sugeno fuzzy nonhomogeneous Markovian jump systems," International Journal of Systems Science, vol. 48, no. 7, pp. 1416-1427, 2017.

[10] Y. Zhang, S. Xu, and B. Zhang, "Robust output feedback stabilization for uncertain discrete-time fuzzy Markovian jump systems with time-varying delays," IEEE Transaction on Fuzzy System, vol. 17, no. 2, pp. 411-420, 2009.

[11] S. He, "Non-fragile passive controller design for nonlinear Markovian jumping systems via observer-based controls," Neurocomputing, vol. 147, pp. 350-357, 2015.

[12] R. Kavikumar, R. Sakthivel, O. M. Kwon, and B. Kaviarasan, "Reliable non-fragile memory state feedback controller design for fuzzy Markov jump systems," Nonlinear Analysis: Hybrid Systems, vol. 35, p. 100828, 2020.

[13] R. Sakthivel, S. Harshavarthini, R. Kavikumar, and Y.-K. Ma, "Robust tracking control for fuzzy Markovian jump systems with time-Varying delay and disturbances," IEEE Access, vol. 6, pp. 66861-66869, 2018.

[14] H. Wang, Y. Jiang, and S. Yin, “Adaptive fuzzy fault tolerant control for Markov jump systems with additive and multiplicative actuator faults," IEEE Transaction on Fuzzy System 1 page, 2020.

[15] H. Yang, Y. Jiang, and S. Yin, "fault-tolerant control of timedelay markov jump systems with $\$$ It $\backslash$ hat $\{0\}$ stochastic process and output disturbance based on sliding mode observer," IEEE Transactions on Industrial Informatics, vol. 14, no. 12, pp. 5299-5307, 2018.

[16] B. Wang and F. C. Zou, "Fault detection filter design for continuous-time nonlinear Markovian jump systems with mode-dependent delay and time-varying transition probabilities," Advances in Differential Equation, vol. 262, pp. 1-23, 2017.

[17] S.-P. He, "Fault estimation for T-S fuzzy Markovian jumping systems based on the adaptive observer," International Journal of Control, Automation and Systems, vol. 12, no. 5, pp. 977-985, 2014.

[18] L. Chen, X. Huang, and S. Fu, "Fault-tolerant control for Markovian jump delay systems with an adaptive observer approach," Circuits, Systems, and Signal Processing, vol. 35, no. 12, pp. 4290-4306, 2016.

[19] F. You, S. Cheng, X. Zhang, and N. Chen, "Robust fault estimation for Takagi-Sugeno fuzzy systems with state timevarying delay," International Journal of Adaptive Control and Signal Processing, vol. 34, no. 2, pp. 141-150, 2020.

[20] F. You, H. Li, F. Wang, and S. Guan, "Robust fast adaptive fault estimation for systems with time-varying interval delay," Journal of the Franklin Institute, vol. 352, no. 12, pp. 54865513, 2015.

[21] K. Zhang, B. Jiang, and P. Shi, "A new approach to observerbased fault-tolerant controller design for Takagi-Sugeno fuzzy systems with state delay," Circuits, Systems and Signal Processing, vol. 28, no. 5, pp. 679-697, 2009.

[22] H. Li, F. Q. You, F. L. Wang, and S. P. Guan, "Robust fast adaptive fault estimation and tolerant control for T-S fuzzy systems with interval time-varying delay," International Journal of System Sciences, vol. 48, no. 8, pp. 1-23, 2017.

[23] H. Gassara, A. El Hajjaji, and M. Chaabane, "Adaptive fault tolerant control design for Takagi-Sugeno fuzzy systems with interval time-varying delay," Optimal Control Applications and Methods, vol. 35, no. 5, pp. 609-625, 2014.

[24] M. Liu and P. Shi, "Sensor fault estimation and tolerant control for Itô stochastic systems with a descriptor sliding mode approach," Automatica, vol. 49, no. 5, pp. 1242-1250, 2013.

[25] Z. Gao and S. X. Ding, "Actuator fault robust estimation and fault-tolerant control for a class of nonlinear descriptor systems," Automatica, vol. 43, no. 5, pp. 912-920, 2007.

[26] M. Liu, P. Shi, L. Zhang, and X. Zhao, "Fault-tolerant control for nonlinear Markovian jump systems via proportional and derivative sliding mode observer technique," IEEE Transactions on Circuits and Systems I: Regular Papers, vol. 58, no. 11, pp. 2755-2764, 2011.

[27] S.-J. Huang and G.-H. Yang, "Fault estimation for a class of linear parameter varying systems with Markovian jumps," Journal of the Franklin Institute, vol. 353, no. 17, pp. 46804700, 2016.

[28] D. Huang and S. K. Nguang, "Robust fault estimator design for uncertain networked control systems with random time delays: an ILMI approach," Information Sciences, vol. 180, no. 3, pp. 465-480, 2010.

[29] T. Youssef, M. Chadli, H. R. Karimi, and R. Wang, "Actuator and sensor faults estimation based on proportional integral observer for TS fuzzy model," Journal of the Franklin Institute, vol. 354, no. 6, pp. 2524-2542, 2017.

[30] Y. Ma, M. Shen, H. Du, Y. Ren, and G. Bian, "An iterative observer-based fault estimation for discrete-time T-S fuzzy systems," International Journal of Systems Science, vol. 51, no. 6, pp. 1007-1018, 2020.

[31] S. Pourdehi and P. Karimaghaee, "Reset observer-based fault tolerant control for a class of fuzzy nonlinear time-delay systems," Journal of Process Control, vol. 85, pp. 65-75, 2020.

[32] H. Gao, Z. Fei, J. Lam, and B. Du, "Further results on exponential estimates of Markovian jump systems with modedependent time-varying delays," IEEE Transactions on Automatic Control, vol. 56, no. 1, pp. 223-229, 2011.

[33] Z. Fei, H. Gao, and P. Shi, "New results on stabilization of Markovian jump systems with time delay," Automatica, vol. 45, no. 10, pp. 2300-2306, 2009.

[34] J. Wu, T. Chen, and L. Wang, "Delay-dependent robust stability and control for jump linear systems with delays," Systems and Control Letters, vol. 55, no. 11, pp. 939-948, 2006. 
[35] X. Zhao, M. Ling, and Q. Zeng, "Delay-dependent stability criterion and $H_{\infty}$ analysis for Markovian jump systems with time-varying delays," Asian Journal of Control, vol. 13, no. 2, pp. 1-8, 2011.

[36] S. K. Nguang, P. Shi, and S. Ding, "Delay-dependent fault estimation for uncertain time-delay nonlinear systems: an LMI approach," International Journal of Robust Nonlinear Control, vol. 16, no. 18, pp. 913-933, 2006.

[37] M. Zhong, S. X. Ding, and Q. Han, "Parity space-based fault estimation for linear discrete time-varying systems," IEEE Trans. on Automatic Control, vol. 55, no. 7, pp. 1726-1731, 2010.

[38] R. Saravanakumar, M. S. Ali, and H. R. Karimi, "Robust Ho control of uncertain stochastic Markovian jump systems with mixed time-varying delays," International Journal of Systems Science, vol. 48, no. 4, pp. 862-872, 2017.

[39] S. He and F. Liu, "Resilient fault detection observer design of fuzzy Markovian jumping systems with mode-dependent time-varying delays," Journal of the Franklin Institute, vol. 353, no. 13, pp. 2943-2965, 2016.

[40] R. Kavikumar, R. Sakthivel, B. Kaviarasan, O. M. Kwon, and S. Marshal Anthoni, "Non-fragile control design for intervalvalued fuzzy systems against nonlinear actuator faults," Fuzzy Sets and Systems, vol. 365, pp. 40-59, 2019.

[41] Y. Ma and M. Chen, "Non-fragile $\mathrm{H} \infty$ state feedback control for singular Markovian jump fuzzy systems with interval time-delay," International Journal of Machine Learning and Cybernetics, vol. 8, no. 4, pp. 1223-1233, 2017. 